\title{
POINCARÉ'S PROBLEM AND THE LENGTH OF THE SHORTEST CLOSED GEODESIC ON A CONVEX HYPERSURFACE
}

\author{
CHRISTOPHER B. CROKE
}

\section{Introduction}

In this paper we discuss two different but related problems.

The first problem is that of finding upper and lower bounds on the length $L$ of the shortest nontrivial closed geodesic on a convex hypersurface $M^{n} \subset \mathbf{R}^{n+1}$. We show that if $M$ encloses a ball of radius $r_{0}$, then $L \geqslant 2 \pi r_{0}$ (Theorem 1.5). We also show (Theorem 1.7) that

$$
L \leqslant \frac{2 \pi}{\sqrt{n} \alpha(n)} \int_{M} \sqrt{S_{n-1}\left(a_{1}^{2}(x), \cdots, a_{n}^{2}(x)\right)} d x,
$$

where $\alpha(n)$ is the volume of the unit $n$ sphere, $\left\{a_{i}(x)\right\}$ is the set of principal curvatures of $M$ at $x$, and $S_{n-1}$ is the $(n-1)$ st symmetric polynomial. Further, if equality holds (in the upper bound), then $M$ is a round sphere. The upper bound is interesting in that it is in terms of an integral of curvatures rather than bounds on curvatures. The lower bound is used in the proof of the second problem.

The second problem was posed by H. Poincaré, in 1905, in a well-known paper [6]. In [6] it was suggested that one could find the shortest simple closed geodesic on a convex surface $M$ by minimizing the arclength functional over the set $\mathcal{Q}$ of all simple smooth closed curves which separate $M$ into two pieces of equal total curvature. Here we establish that this suggestion in fact works.

In 1980 using the methods of integral currents M. S. Berger and E. Bombieri [1] showed that the result holds for metrics $C^{3}$ close to the standard metric. The reason for this restriction comes in showing that the minimum which they get is connected. They suggest that by complicating the proofs and using the theory of varifolds one may be able to extend their proof to cover all convex surfaces. 
In this paper, we take a completely different approach. We consider compact finite dimensional approximating spaces $\Omega_{1 / 2}^{m}(M)$ of closed piecewise geodesic curves. The main difficulty with this approach is to show that the minima achieved are simple.

One should note that if $\gamma$ is a nontrivial simple closed geodesic on $M$, then by Gauss-Bonnet $\gamma$ splits $M$ into two pieces of equal total curvature so $\gamma \in \mathbb{Q}$. On the other hand the first variation formula (see [1, §2]) shows that if $\tau \in \mathbb{Q}$ and the length of $\tau$ is the infimum $\tilde{L}$ of the length functional on $\mathcal{Q}$, then $\tau$ is a simple closed geodesic. Thus we will show (Theorem 3.2) that there is a simple closed geodesic $\gamma$ with length $\tilde{L}$. (The fact that $\tilde{L}>0$ will follow from Corollary 1.4.)

In the first section we prove the upper and lower bounds on the length of the shortest nontrivial closed geodesic.

In the second section we define the approximating spaces $\Omega_{1 / 2}^{m}(M)$ and state some properties of these spaces. Unfortunately, although most of the properties are geometrically intuitive, the proofs are often long and tedius. Therefore the proofs of the lemmas in $\$ 2$ are included in the appendix at the end of the paper.

In the third section we show that Poincaré's suggestion works.

The author would like to thank Wolfgang Ziller and Werner Ballmann for bringing the Poincaré problem to his attention. He would also like to thank Eugenio Calabi for helpful conversations.

\section{The length of the shortest closed geodesic on a convex hypersurface}

In this section we prove an upper and lower bound on the length of the shortest closed geodesic on a convex hypersurface $M^{n} \subset \mathbf{R}^{n+1}$. In the process we will derive a result, Corollary 1.4 , which will be needed in the proof of Poincarés problem.

We begin with two lemmas.

Lemma 1.1. Let $M \subset \mathbf{R}^{n+1}$ be a convex hypersurface which encloses the ball of radius $r_{0}$, centered at the origin. Then for all $x \in M$ we have $\langle x, N(x)\rangle \geqslant r_{0}$ where $N(x)$ is the image of $x$ under the gauss map (i.e., $N(x)$ is the unit outward normal to $M$ at $x$ ). Further equality holds if and only if $x$ lies on the sphere of radius $r_{0}$.

Proof. $\langle x, N(x)\rangle$ is the distance from the origin to the tangent plane $P$ of $M$ at $x$. Since $M$ is convex, $P$ lies outside $M$, hitting $M$ only at $x$. Hence $P$ lies outside the sphere of radius $r_{0}$, hitting the sphere only if $x$ lies on the sphere. 
Lemma 1.2. Let $\gamma$ be a closed piecewise $C^{1}$ curve in $\mathbf{R}^{n+1}$ of length $L$. Then there is a point $x_{0} \in \mathbf{R}^{n+1}$ such that for all $t,\left|x_{0}-\gamma(t)\right| \leqslant L / 4$. Further we may choose $x_{0}$ so that the inequality is strict unless $\gamma$ is a line segment of length $L / 2$ transversed twice.

Proof. Let $\gamma:[0, L] \rightarrow M$ be parameterized by arclength. Let $x_{0}=\frac{1}{2}(\gamma(0)$ $+\gamma(L / 2)$ ) (i.e., $x_{0}$ is the midpoint of the line segment between $\gamma(0)$ and $\gamma(L / 2))$. Now for all $t \in[0, L]$

$$
\begin{aligned}
2\left|x_{0}-\gamma(t)\right| & =\left|\left(2 x_{0}-\gamma(t)\right)-\gamma(t)\right| \\
& \leqslant\left|\left(2 x_{0}-\gamma(t)\right)-\gamma(0)\right|+|\gamma(0)-\gamma(t)| \\
& =|\gamma(L / 2)-\gamma(t)|+|\gamma(0)-\gamma(t)| \\
& \leqslant L / 2 .
\end{aligned}
$$

It is not hard to see that if equality holds above for some $t_{0} \in[0, L / 2]$, then $\gamma_{[0, L / 2]}$ is the line segment from $\gamma(0)$ to $\gamma\left(t_{0}\right)$ followed by the line segment from $\gamma\left(t_{0}\right)$ to $\gamma(L / 2)$ and that these line segments lie on the same line (see the first inequality). If equality does not also hold for some $t_{1} \in[L / 2, L]$, then one could make the inequality strict by moving $x_{0}$ slightly. The above shows that $\gamma$ must be the line segment from $\gamma\left(t_{0}\right)$ to $\gamma\left(t_{1}\right)$ transversed twice except in the case where $x_{0}=\gamma(0)=\gamma(L / 2)$ and the line segments from $x_{0}$ to $\gamma\left(t_{0}\right)$ and $x_{0}$ to $\gamma\left(t_{1}\right)$ make an angle at $x_{0}$. In this case we could again move $x_{0}$ slightly to achieve strict inequality.

Theorem 1.3. Let $M^{n}$ be a convex hypersurface in $\mathbf{R}^{n+1}$ which enclose a ball of radius $r_{0}$. Then every closed piecewise $C^{1}$ curve $\gamma$ on $M$, whose image under the gauss map hits every closed hemisphere of the unit sphere, has length greater than $4 r_{0}$.

Proof. We may assume that the origin is the center of the ball of radius $r_{0}$. Let $x_{0}$ be as in Lemma 1.2. There are two cases.

Case 1. $x_{0}=0$. Since $\gamma(t)$ lies on $M$ which encloses the ball of radius $r_{0}$, we have $r_{0} \leqslant|\gamma(t)|=\left|\gamma(t)-x_{0}\right| \leqslant L / 4$. Now if equality holds in the first inequality for all $t$, then $\gamma$ lies on the sphere of radius $r_{0}$. But in this case Lemma 1.2 says equality cannot hold in the second inequality, so $L>4 r_{0}$.

Case 2. $x_{0} \neq 0$. Since the image of $\gamma$ under the gauss map hits every closed hemisphere, there is a $t$ such that $\left\langle x_{0}, N(\gamma(t))\right\rangle \leqslant 0$. Thus

$$
L / 4 \geqslant\left|\gamma(t)-x_{0}\right| \geqslant\left\langle\gamma(t)-x_{0}, N(\gamma(t))\right\rangle \geqslant\langle\gamma(t), N(\gamma(t))\rangle \geqslant r_{0},
$$

so $L \geqslant 4 r_{0}$.

If equality holds, then Lemma 1.1 says that $\gamma(t)$ lies on the sphere of radius $r_{0}$, and since $\langle\gamma(t), N(\gamma(t))\rangle=r_{0}, \gamma(t)=r_{0} N(\gamma(t))$. So $\left\langle x_{0}, \gamma(t)\right\rangle=0$, and $x_{0} \neq 0$ shows that $\left|\gamma(t)-x_{0}\right|>r_{0}$. Thus equality never holds and $L>4 r_{0}$. 
Example. The following example shows that $4 r_{0}$ is the best possible lower bound in Theorem 1.3. One might suspect at first that the lower bound is $2 \pi r_{0}$, as this is the lower bound in the case that $M$ is the sphere.

Let $\gamma$ be the ellipse in $\mathbf{R}^{3}$ described by $z=2, x^{2} /(1+\varepsilon)^{2}+y^{2} / \varepsilon^{2}=1$ for some small $\varepsilon>0$. Let $K$ be the convex hull of $\gamma$ and the unit sphere centered at the origin. By perturbing $K$ slightly below $z=2$ and rounding off above $z=2$, we can construct a smooth convex two-manifold $M$ enclosing $K$ such that $M$ is symmetric with respect to $x$ and $y, \gamma \subset M$, and the $z$ component of the normal is positive at $(0, \varepsilon, 2)$ and $(0,-\varepsilon, 2)$, and negative at $(1+\varepsilon, 0,2)$ and $(-1-$ $\varepsilon, 0,2)$.

If $t$ is a parameter for $\gamma:[0,1] \rightarrow M$ which is symmetric with respect to $x$ and $y$ (i.e., $d t \rightarrow-d t$ under $x \rightarrow-x$ and under $y \rightarrow-y$ ) and $N(\gamma(t))=$ $(x(t), y(t), z(t))$, then $\int_{0}^{1} x(t) d t=\int_{0}^{1} y(t) d t=0$ by the symmetry. Further, since $z(t)<0$ at some $t$ and $z(t)>0$ for some $t$, we can choose a symmetric $t$ such that $\int_{0}^{1} z(t)=0$. With this parameter $\int_{0}^{1} N(\gamma(t))=0$, and hence we see that the curve $N(\gamma(t))$ hits every hemisphere.

To finish the example one needs only note that as $\varepsilon$ goes to 0 the length of $\gamma$ approaches 4 .

Corollary 1.4. Let $M$ be a convex surface in $\mathbf{R}^{3}$, and $\gamma$ a closed piecewise $C^{1}$ curve on $M$ such that $M-\gamma$ consists of two open sets $M^{+}$and $M^{-}$(not necessarily connected, but of course the union of connected components) of equal total curvature. Then the length of $\gamma$ is greater than $4 r_{0}$.

Proof. Since $M$ is convex, the gauss map $G: M \rightarrow S^{2}$, where $S^{2}$ is the unit sphere, is a diffeomorphism. Now the volume of $G\left(M^{+}\right)$(and $G\left(M^{-}\right)$) is $2 \pi$, the total curvature of $M^{+}$(and $M^{-}$). Hence the curve $N(\gamma(t))$ hits every closed hemisphere, and the theorem gives the result.

Remarks. In some special cases one is able to improve the constant $4 r_{0}$ in Theorem 1.3 to the more natural constant $2 \pi r_{0}$. In [4] it was shown that this is the case if $\gamma$ is a closed $C^{1}$ curve on $M$ such that $\int_{0}^{L} N(\gamma(s)) d s=0$, where $s$ is the arclength parameter. Another case is the following theorem.

Theorem 1.5. Let $M^{n} \subset \mathbf{R}^{n+1}$ be a convex hypersurface enclosing a ball of radius $r_{0}$, and let $\gamma \subset M$ be a nontrivial closed geodesic. Then $L(\gamma) \geqslant 2 \pi r_{0}$, with equality holding if and only if $\gamma$ is a great circle on the sphere of radius $r_{0}$.

Proof. Let $s$ be the arclength parameter for $\gamma$, and $k(s)$ be the curvature of $\gamma$ as a space curve. Since $\gamma$ is a geodesic on $M, \gamma^{\prime \prime}(s)=-k(s) N(\gamma(s))$ where $N(\gamma(s))$ is the unit outward normal to $M$ at $\gamma(s)$. Thus

$$
\begin{aligned}
L(\gamma) & =\int_{0}^{L(\gamma)}\left|\gamma^{\prime}(s)\right|^{2} d s=\int_{0}^{L(\gamma)} \frac{d}{d s}\left\langle\gamma(s), \gamma^{\prime}(s)\right\rangle d s-\int_{0}^{L(\gamma)}\left\langle\gamma(s), \gamma^{\prime \prime}(s)\right\rangle d s \\
& =\int_{0}^{L(\gamma)}\left\langle\gamma(s),-\gamma^{\prime \prime}(s)\right\rangle d s=\int_{0}^{L(\gamma)} k(s)\langle\gamma(s), N(\gamma(s))\rangle d s .
\end{aligned}
$$


By Lemma 1.1, $\langle\gamma(s), N(\gamma(s))\rangle \geqslant r_{0}$. So

$$
L(\gamma) \geqslant r_{0} \int_{0}^{L(\gamma)} k(s) d s \geqslant 2 \pi r_{0}
$$

If the equality holds in the above formula, then it also holds in Lemma 1.1, so $\gamma$ lies on the sphere of radius $r_{0}$. To see that $\gamma$ is a geodesic on this sphere, we need only note that at $\gamma(s)$ the tangent space to the sphere and the tangent space to $M$ coincide, so $\gamma^{\prime \prime}(s)$ is perpendicular to the sphere.

We now prove that the length of the shortest closed geodesic on a convex hypersurface has an upper bound which is an integral of a function of the principal curvatures.

Let $M^{n} \subset \mathbf{R}^{n+1}$ be a convex hypersurface, and $G: M^{n} \rightarrow S^{n}$ be the gauss map. Since $M$ is convex, $G$ is a diffeomorphism. If $\gamma$ is a geodesic (a great circle) on $S^{n}$, then the smooth closed curve $G^{-1}(\gamma)$ on $M$ is called a shadow curve.

Lemma 1.6. Let $L$ be a length of the shortest closed geodesic on a convex hypersurface $M$, and $\bar{L}$ the length of the shortest shadow curve. Then $\bar{L} \geqslant L$.

Proof. Let $\gamma$ be a great circle on $S^{n}$ such that $L\left(G^{-1}(\gamma)\right)=\bar{L}$. Let $P$ be the plane in $\mathbf{R}^{n+1}$ determined by $\gamma$.

We claim that if $\bar{P}$ is any plane parallel to $P$, then the length of $\bar{P} \cap M$ is less than or equal to $\bar{L}$. To see this, let $\pi: \mathbf{R}^{n+1} \rightarrow P$ be the orthogonal projection. $\pi(\vec{P} \cap M)$ is a convex curve $\tau$ in $P$, which is contained in the convex region $\pi(M)$, and further $L(\tau)=L(\bar{P} \cap M)$. By the definition of the gauss map, the boundary of $\pi(M)$ is precisely $\pi\left(G^{-1}(\gamma)\right)$. Hence $\bar{L}=$ $L\left(G^{-1}(\gamma)\right) \geqslant L\left(\pi\left(G^{-1}(\gamma)\right)\right) \geqslant L(\tau)=L(\bar{P} \cap M)$, and the claim follows.

Let $P^{\perp}$ be the $(n-1)$-dimensional plane in $\mathbf{R}^{n+1}$ perpendicular to $P$, and let $S \subset P^{\perp}$ be the orthogonal projection of $M$ to $P^{\perp}$. For $x \in S$, let $\gamma_{x}$ be $\bar{P}_{x} \cap M$, where $\bar{P}_{x}$ is the plane parallel to $P$ through $x$. Let $C_{x} \in \bar{P}_{x}$ be the center of mass of $\gamma_{x}$. Fix an orthonormal basis $e_{1}, e_{2}$ for $P$, and let $e_{1}^{x}, e_{2}^{x}$ be the parallel translate of this basis to $C_{x}$. For $t \in[0,2 \pi]$ let $r_{x}^{t}$ be the ray in $\bar{P}_{x}$ from $C_{x}$ making an angle $t$ with $e_{1}^{x}$ where the angle is determined such that $r_{x}^{\pi / 2}$ corresponds to $e_{2}^{x}$. We now parameterize $\gamma_{x}$ such that $\gamma_{x}(t)=\gamma_{x} \cap r_{x}^{t}$, if $\gamma_{x}$ is not trivial, and parameterize the trivial curves in the only way possible. This construction clearly makes the parameter vary continuously with $x$.

Thus we have constructed a continuous map from $S$ to $\Omega(M)$, the free loop space of $M$. Let $\Omega_{0}(M)$ represent the trivial loops on $M$. Then the map $x \rightarrow \gamma_{x}$ gives rise to a homotopically nontrivial map of $S^{n-1}$ to $\Omega(M) / \Omega_{0}(M)$. Now a standard minimax procedure (see [3]) gives rise to a closed geodesic $\sigma$ on $M$ such that $L(\sigma) \leqslant \max _{x \in s} L\left(\gamma_{x}\right)$. 
Hence combining this with the claim we have

$$
\bar{L} \geqslant \max _{x \in s} L\left(\gamma_{x}\right) \geqslant L(\sigma) \geqslant L .
$$

Theorem 1.7. Let $L$ be the length of the shortest nontrivial closed geodesic on a convex hypersurface $M^{n} \subset \mathbf{R}^{n+1}$. For $x \in M$ let $a_{1}(x), a_{2}(x), \cdots, a_{n}(x)$ be the principal curvatures of $M$ at $x$. Then

$$
L \leqslant \frac{2 \pi}{\alpha(n) \sqrt{n}} \int_{M} \sqrt{S_{n-1}\left(a_{1}^{2}(x), a_{2}^{2}(x), \cdots, a_{n}^{2}(x)\right)} d x,
$$

where $\alpha(n)$ represents the volume of the unit $n$-sphere, and $S_{n-1}$ the $(n-1)$ st symmetric polynomial. In particular, for $n=2$ we have

$$
L \leqslant \frac{1}{2 \sqrt{2}} \int_{M} \sqrt{a_{1}^{2}(x)+a_{2}^{2}(x)} d x .
$$

Further, if equality holds, then $M$ is a round sphere.

Proof. Let $G: M \rightarrow S^{n}$ be the gauss map. Let $\Gamma$ be the space of great circles on $S^{n}$ with the usual measure, that is, the measure such that for any function $f$ : $U S^{n} \rightarrow \mathbf{R}$, where $U S^{n}$ is the unit sphere bundle, we have

$$
\int_{U S^{n}} f(v) d v=\int_{\Gamma} \int_{0}^{2 \pi} f\left(\gamma^{\prime}(t)\right) d t d \gamma
$$

In particular, $\operatorname{Vol}(\Gamma)=\frac{1}{2 \pi} \alpha(n) \alpha(n-1)$.

Let $g(\cdot, \cdot)$ represent the metric on $M$. Then by Lemma 1.7 we have

(i)

$$
\begin{aligned}
L & \leqslant \frac{1}{\operatorname{vol}(\Gamma)} \int_{\Gamma} L\left(G^{-1}(\gamma)\right) d \gamma \\
& =\frac{2 \pi}{\alpha(n) \alpha(n-1)} \int_{\Gamma} \int_{0}^{2 \pi} \sqrt{g\left(G_{*}^{-1}\left(\gamma^{\prime}(t)\right), G_{*}^{-1}\left(\gamma^{\prime}(t)\right)\right)} d t d \gamma \\
& =\frac{2 \pi}{\alpha(n) \alpha(n-1)} \int_{U S^{n}} \sqrt{\left(\left(G^{-1}\right)^{*} g\right)(v, v)} d v \\
& =\frac{2 \pi}{\alpha(n) \alpha(n-1)} \int_{S^{n}}\left[\int_{U_{P}} \sqrt{\left(\left(G^{-1}\right)^{*} g\right)(v, v)} d v\right] d p
\end{aligned}
$$

where $U_{p}$ is the unit tangent sphere at $p \in S^{n}$.

Now we have

(ii) $\int_{U_{p}} \sqrt{\left(\left(G^{-1}\right)^{*} g\right)(v, v)} d v \leqslant \sqrt{\int_{U_{p}}\left(\left(G^{-1}\right)^{*} g\right)(v, v) d v} \sqrt{\alpha(n-1)}$, 
(iii)

$$
\begin{aligned}
\int_{U_{p}}\left(\left(G^{-1}\right)^{*} g\right)(v, v) d v & =\frac{\alpha(n-1)}{n} \operatorname{tr}\left(\left(G^{-1}\right)^{*} g\right)(p) \\
& =\frac{\alpha(n-1)}{n} \sum_{i=1}^{n} \frac{1}{a_{i}^{2}\left(G^{-1}(p)\right)} .
\end{aligned}
$$

Combining (i), (ii), and (iii) we get

$$
\begin{aligned}
L & \leqslant \frac{\pi}{\alpha(n) \sqrt{n}} \int_{S^{n}}\left[\sum_{i=1} a_{i}^{2}\left(G^{-1}(p)\right)\right]^{-1 / 2} d p \\
& =\frac{2 \pi}{\alpha(n) \sqrt{n}} \int_{M}\left[\sum_{i=1}^{n} a_{i}^{2}(x)\right]^{-1 / 2} a_{1}(x), \cdots, a_{n}(x) d x \\
& =\frac{2 \pi}{\alpha(n) \sqrt{n}} \int_{M} \sqrt{S_{n-1}\left(a_{1}^{2}(x), \cdots, a_{n}^{2}(x)\right)} d x
\end{aligned}
$$

It is clear that equality holds at each step for a round sphere. On the other hand, in order for equality to hold we must have $L=L\left(G^{-1}(\gamma)\right)$ for every great circle $\gamma$. Tracing through equality in Lemma 1.6 we see that $G^{-1}(\gamma)$ is a closed geodesic for each $\gamma$. Hence $M$ is a Blaske sphere and is thus, by the theorem of Berger, Kazdan, Weinstein, and Yang (see [2, Appendices D and E]), isometric to a round sphere.

Corollary 1.8. Let $M$ and $L$ be as in Theorem 1.7. Then the following hold:

(a) $L \leqslant 2 \pi / \alpha(n) \int_{M} a_{2}(x) \cdots a_{n}(x) d x$ where $a_{1}(x)$ is the smallest principal curvature at $x$. Equality holds if and only if $M$ is a round sphere.

(b) $L<2 \pi / \alpha(n) \sqrt{n} \int_{M} S_{n-1}\left(a_{1}(x), \cdots, a_{n}(x)\right) d x$.

In particular for $n=2$

$$
L<\frac{1}{\sqrt{2}} \int_{M} H(x) d x,
$$

where $H(x)$ is the mean curvature of $M$ at $x$.

Proof. This follows directly from the theorem and the fact that $a_{i}(x)>0$ for all $x \in M$ and $i=1,2, \cdots, n$.

\section{The approximating spaces $\Omega_{1 / 2}^{m}(M)$}

We let $M$ be a given convex surface, and $c$ be $1 / 3$ the convexity radius of $M$. For fixed $m$ the most natural approximating space would be the closure of the set of all simple closed piecewise geodesics with $m$ breaks which split $M$ into two pieces of equal total curvature and have the length of each segment less 
than or equal to $c$. However, in order to simplify the (nevertheless tedious) proofs we define $\Omega_{1 / 2}^{m}(M)$ in a more complicated way.

We begin by defining a larger space.

Definition. $\Omega^{m}(M)$ is the set of all closed curves $\gamma:[0,1] \rightarrow M(\gamma(0)=\gamma(1))$ satisfying the following conditions:

(a) If $\gamma^{n}$ is $\gamma_{\left[\frac{n-1}{m}, \frac{n}{m}\right]}$, then $\gamma^{n}:\left[\frac{n-1}{m}, \frac{n}{m}\right] \rightarrow M$ is a geodesic segment (parameterized proportional to arc length).

(b) Let $l_{n}$ be the length of $\gamma^{n}$. Then $l_{n} \leqslant c$.

(c) $\gamma$ is the limit of simple piecewise smooth closed curves. That is, for every $\varepsilon>0$ there is a simple piecewise smooth closed curve $c_{\varepsilon}^{\gamma}:[0,1] \rightarrow M$ such that for all $t \in[0,1]$ we have $d\left(\gamma(t), c_{\varepsilon}^{\gamma}(t)\right)<\varepsilon$, where $d$ represents the distance in M.

Although we will speak of $\gamma$ as a map from $[0,1]$ we think of it as a map from $S^{1}$ in the usual way. In particular when we speak of the parameter $t$ or the points $n / m$ we mean them modulo the integers in the usual way.

The topology on $\Omega^{m}(M)$ is the one induced by the embedding $\Omega^{m}(M)$ $\rightarrow M \underbrace{\times \ldots \times}_{m \text { times }} M$ where $\gamma$ is mapped to $(\gamma(1 / m), \gamma(2 / m), \ldots, \gamma(1))$.

The energy $E$ and length $L$ functionals are given by

$$
L(\gamma)=\sum_{i=1}^{m} l_{i}, \quad E(\gamma)=m \sum_{i=1}^{m}\left(l_{i}\right)^{2}
$$

(Notice we do not include the usual factor of $1 / 2$ in $E$.)

Another useful parameter for $\gamma \in \Omega^{m}(M)$ is the arclength parameter $s$. If $\gamma \in \Omega^{m}(M)$ is such that $l_{i}>0$ for all $i$, then the parameter $t$ can be thought of as a function of $s$, that is, $\gamma(t(s))$ is the same curve only with the arclength parameter.

Lemma 2.1. $\Omega^{m}(M)$ is compact and contains the simple curves $\gamma$ which satisfy conditions (a) and (b) of the definition.

Proof. To see that $\Omega^{m}(M)$ is compact let $\gamma_{i} \rightarrow \gamma$ in $M \times \cdots \times M$. Clearly conditions (a) and (b) are satisfied by $\gamma$. To check condition (c), fix $\varepsilon>0$ and choose $i$ so large that $d\left(\gamma_{i}(t), \gamma(t)\right)<\varepsilon / 2$. Consider the curve $C_{\varepsilon / 2}^{\gamma_{i}}$ which exists since $\gamma_{i} \in \Omega^{m}(M)$. We then have

$$
d\left(\gamma(t), C_{\varepsilon / 2}^{\gamma_{i}}(t)\right) \leqslant d\left(\gamma(t), \gamma_{i}(t)\right)+d\left(\gamma_{i}(t), C_{\varepsilon / 2}^{\gamma_{i}}(t)\right)<\varepsilon,
$$

hence $\gamma$ satisfies property (c).

The other part of the lemma follows from the fact that any simple curve satisfying (a) and (b) automatically satisfies (c), i.e., just let $C_{\varepsilon}^{\gamma}$ be $\gamma$ itself. 
Lemma 2.2. If $\gamma \in \Omega^{m}(M)$ is such that for some $i, l_{i}=0$ and $l_{i+1} \neq 0$, let

$$
\bar{\gamma}(t)= \begin{cases}\gamma(t) & \text { if } t \notin\left[\frac{i-1}{m}, \frac{i+1}{m}\right], \\ \gamma\left(\frac{1}{2}\left(t-\frac{i-1}{m}\right)+\frac{i}{m}\right) & \text { if } t \in\left[\frac{i-1}{m}, \frac{i+1}{m}\right],\end{cases}
$$

Then

(a) $\bar{\gamma} \in \Omega^{m}(M)$,

(b) $\bar{l}_{i} \neq 0, \overline{l_{i+1}} \neq 0, E(\bar{\gamma})<E(\gamma)$, and $L(\bar{\gamma})=L(\gamma)$,

(c) $\gamma$ is homotopic to $\bar{\gamma}$ inside $\gamma[0,1]$.

Proof. See appendix.

A curve $\gamma \in \Omega^{m}(M)$ is said to make a $180^{\circ}$ turn at $i / m$ if $l_{i} \neq 0, l_{i+1} \neq 0$ and $\gamma\left(i / m-t / m l_{i}\right)=\gamma\left(i / m+t / m l_{i+1}\right)$ for all $0 \leqslant t \leqslant \min \left\{l_{i}, l_{i+1}\right\}$.

Lemma 2.3. Let $\gamma \in \Omega^{m}(M)$ be such that $l_{j} \neq 0$ for all $j$ and such that $\gamma$ makes a $180^{\circ}$ turn at $i / m$ for some $i$. Then there is a curve $\bar{\gamma}$ such that

(a) $\bar{\gamma} \in \Omega^{m}(M)$,

(b) $L(\bar{\gamma})<L(\gamma), E(\bar{\gamma})<E(\gamma)$,

(c) $\bar{\gamma}$ is homotopic to $\gamma$ inside $\gamma([0,1])$.

Proof. See appendix.

For $c:[0,1] \rightarrow M$ a simple closed curve then $M-c$ consists of two open connected components $M^{+}(c)$ and $M^{-}(c)$, where $M^{+}(c)$ is the component for which the orientation of $c$ is the same as the orientation of $\partial M^{+}(c)$.

A curve $\gamma \in \Omega^{m}(M)$ is said to be nondegenerate if there is a $\delta>0$ such that for all sufficiently small $\varepsilon>0$ and for all piecewise smooth simple closed curves $c:[0,1] \rightarrow M$ with $d(c(t), \gamma(t))<\varepsilon$ we have $\operatorname{Vol}\left(M^{+}(c)\right)>\delta$ and $\operatorname{Vol}\left(M^{-}(c)\right)>\delta$.

Definition. Given $\gamma \in \Omega^{m}(M)$ nondegenerate we say that $x$ is in $M^{+}(\gamma)$ if $x \in M-\gamma$ and for all sufficiently small $\varepsilon$ and all piecewise smooth curves $c$ such that $d(\gamma(t), c(t))<\varepsilon$ we have $x \in M^{+}(c)$. Similarly we define $M^{-}(\gamma)$.

Lemma 2.4. For $\gamma \in \Omega^{m}(M)$ nondegenerate, the following are true.

(a) $M^{+}(\gamma) \cup M^{-}(\gamma)=M-\gamma$.

(b) If $K$ is a component of $M-\gamma$, then $K \subset M^{+}(\gamma)$ or $K \subset M^{-}(\gamma)$.

(c) If $f: M \rightarrow \mathbf{R}$ is any continuous function, we have $\int_{M^{+}(\gamma)} f=\lim _{\varepsilon \rightarrow 0} \int_{M^{+}\left(c_{\varepsilon}^{\gamma}\right)} f$. Similarly for $\mathrm{M}^{-}(\gamma)$.

(d) If $x \in M^{+}(\gamma), y \in M^{-}(\gamma)$ and $\bar{\gamma} \in \Omega^{m}(M)$ such that $\bar{\gamma}$ is homotopic to $\gamma$ in $M-\{x, y\}$, then $\bar{\gamma}$ is nondegenerate, and $x \in M^{+}(\bar{\gamma})$ and $y \in M^{-}(\bar{\gamma})$.

Proof. See appendix.

A curve $\gamma \in \Omega^{m}(M)$ is said to be regular if $\gamma$ is nondegenerate, $l_{i}>0$ for all $i$, and $\gamma$ makes no $180^{\circ}$ turns. 
For $\gamma \in \Omega^{m}(M)$ regular and $i=1,2, \cdots, m$ we define $\operatorname{Ext}(i)$ to be the exterior angle at $\gamma(i / m)$ between $\gamma^{i}$ and $\gamma^{i+1}$. Since $\gamma$ makes no $180^{\circ}$ turns, we see $-\pi<\operatorname{Ext}(i)<\pi$. $i$ is called a + vertex if $\operatorname{Ext}(i)>0$, and a - vertex if $\operatorname{Ext}(i)<0$.

Later in this paper we will want to make the deformations indicated in Fig. 1.
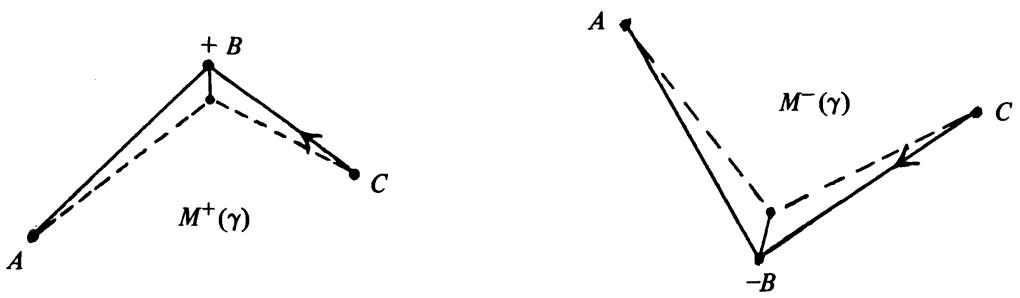

FIG. 1

However, these deformations may not be allowed for a couple of reasons.

The first thing which can go wrong is that the piece of the curve $\gamma$ shown in the figure may be transversed many times by $\gamma$. In this case only the "innermost" parameter piece of $\gamma$ can be so deformed, since the deformation of another parameter piece of $\gamma$ will lead to a curve which is not the limit of simple curves.

The other thing that can go wrong is that another piece of $\gamma$ could prevent the deformation (see Fig. 2).

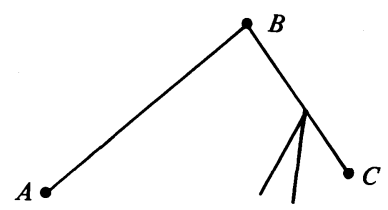

(a)

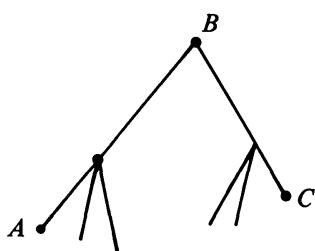

(b)

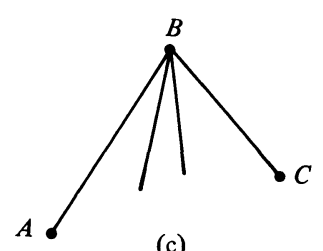

(c)

FIG. 2

One should note that in Fig. 2(a) one can still make a length decreasing deformation (see Fig. 3); however, this deformation may increase energy. This "half" deformation will also be important.

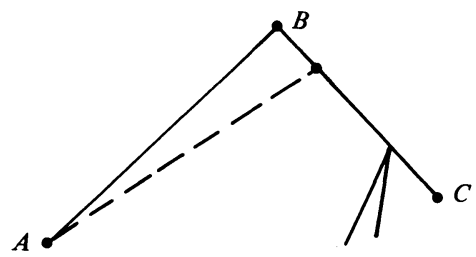

FIG. 3 
We will now be precise about which vertices of $\gamma$ can be so deformed (or half deformed) so that the resulting curve is still the limit of simple curves.

Let $\gamma$ be a regular element of $\Omega^{m}(M)$ and $t_{1}, t_{2} \in[0,1]-$ $\{0,1 / m, 2 / m, \cdots, 1\}$ such that $t_{1} \neq t_{2}$ and $\gamma\left(t_{1}\right)=\gamma\left(t_{2}\right)$. Let $V_{0}$ be the unit tangent vector to $\gamma$ at $t_{1}$. Note that the unit tangent vector to $\gamma$ at $t_{2}$ is either $V_{0}$ or $-V_{0}$ as $\gamma$ cannot have transverse self intersections and still be the limit of simple curves. Let $S_{t_{1}}$ and $S_{t_{2}}$ be the arclength parameters corresponding to $t_{1}$ and $t_{2}$ respectively; i.e., $t\left(S_{t_{1}}\right)=t_{1}$ and $t\left(S_{t_{2}}\right)=t_{2}$ where $\gamma(t(S))$ is $\gamma$ parameterized by arclength.

Let $\left[S_{0}\left(t_{1}, t_{2}\right), S_{1}\left(t_{1}, t_{2}\right)\right]$ be the maximal compact interval $\left(S_{0}\left(t_{1}, t_{2}\right)<0<\right.$ $\left.S_{1}\left(t_{1}, t_{2}\right)\right)$ such that for all $S \in\left[S_{0}\left(t_{1}, t_{2}\right), S_{1}\left(t_{1}, t_{2}\right)\right]$ we have $\gamma\left(t\left(S_{t_{1}}+S\right)\right)=$ $\gamma\left(t\left(S_{t_{2}}+S\right)\right.$ ) (or if the unit tangent vector to $\gamma$ at $t_{2}$ is $-V_{0}$ we have $\left.\gamma\left(t\left(S_{t_{1}}+S\right)\right)=\gamma\left(t\left(S_{t_{2}}-S\right)\right)\right)$. The only thing which needs to be verified here is that the maximal such interval is bounded. We look at the two cases. If the unit tangent vector to $\gamma$ at $t_{2}$ is $V_{0}$, and the length of the interval is greater than the length of $\gamma$, then $\gamma:[0,1] \rightarrow M$ must transverse its image at least twice and hence cannot be the limit of simple curves. If the unit tangent vector to $\gamma$ at $t_{2}$ is $-V_{0}$, and the length of the interval is greater than the length of $\gamma$, then there would be a $180^{\circ}$ turn somewhere in $[0,1]$, but this cannot happen since $\gamma$ is regular.

Given $V$ a unit vector at $\gamma\left(t_{1}\right)=\gamma\left(t_{2}\right)$ perpendicular to $V_{0}$, we will now define an ordering $>V$ on $\left\{t_{1}, t_{2}\right\}$, i.e., either $t_{1}>t_{2}$ or $t_{2}>t_{1}$.

Define the unit vectors $\bar{V}_{0}, \bar{V}, V_{1}, V_{2}$ at the point $\gamma\left(t\left(S_{t_{1}}+S_{1}\left(t_{1}, t_{2}\right)\right)\right)=$ $\gamma\left(t\left(S_{t_{2}} \pm S_{1}\left(t_{1}, t_{2}\right)\right)\right)$ as follows. Let $t_{i}=t\left(S_{t_{i}} \pm S_{1}\left(t_{1}, t_{2}\right)\right)$. Then:

$\bar{V}_{0}$ is tangent to $-\gamma_{\left\langle\left(\bar{t}_{1}-\varepsilon, \overline{t_{1}}\right]\right.}$,

$V_{1}$ is tangent to $\gamma_{\mid\left[\bar{t}_{1}, \bar{t}_{1}+\varepsilon\right)}$

$V_{2}$ is tangent to $\gamma_{\mid\left[\bar{t}_{2}, \bar{t}_{2}+\varepsilon\right)}$ (or to $\gamma_{\left.\mid t_{2}-\varepsilon, \bar{t}_{2}\right]}$ if the unit tangent to $\gamma$ at $t_{2}$ is $-V_{0}$ ),

$\bar{V}$ is the unit vector perpendicular to $V_{0}$ such that the orientation given by $\left(\bar{V}_{0}, \bar{V}\right)$ is the same as that given by $\left(-V_{0}, V\right)$. (See Fig. 4.)

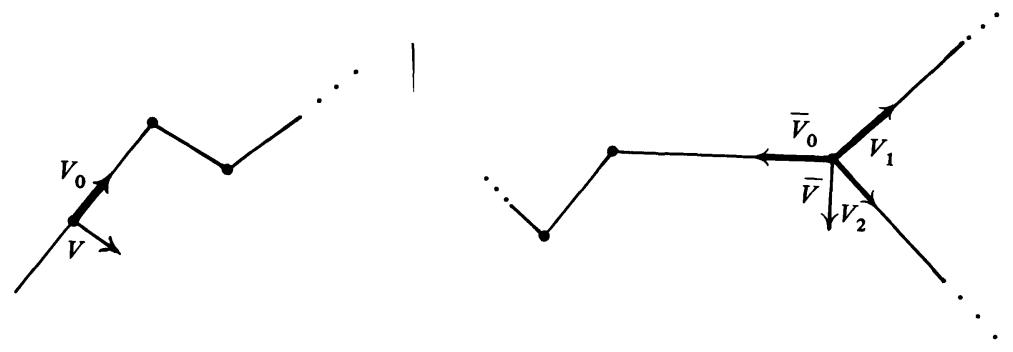

FIG. 4 
We notice that $V_{1} \neq V_{2}$ since equality would contradict the maximality of $\left[S_{0}\left(t_{1}, t_{2}\right), S_{1}\left(t_{1}, t_{2}\right)\right]$. We also notice that $\bar{V}_{0} \neq V_{1}, \bar{V}_{0} \neq V_{2}$ since $\gamma$ has no $180^{\circ}$ turns.

Now measure angles from $\bar{V}_{0}$ (either clockwise or counterclockwise) so that the angle at $\bar{V}$ is $\pi / 2$. We then say that $t_{2}>t_{1}$ if the angle from $\bar{V}_{0}$ to $V_{2}$ is smaller than the angle from $\bar{V}_{0}$ to $V_{1}$. Intuitively $t_{2}>t_{1}$ says that for simple approximating curves the piece corresponding to $t_{2}$ will be more "inner" (with respect to $V$ ) than the piece corresponding to $t_{1}$.

We could have looked instead at unit vectors (the signs appropriately chosen, see Fig. 5) at $\gamma\left(t\left(S_{t_{1}}+S_{0}\left(t_{1}, t_{2}\right)\right)\right.$. The ordering on $t_{1}, t_{2}$ would be the same for if not (see Fig. 5), $\gamma$ could not be the limit of simple curves.
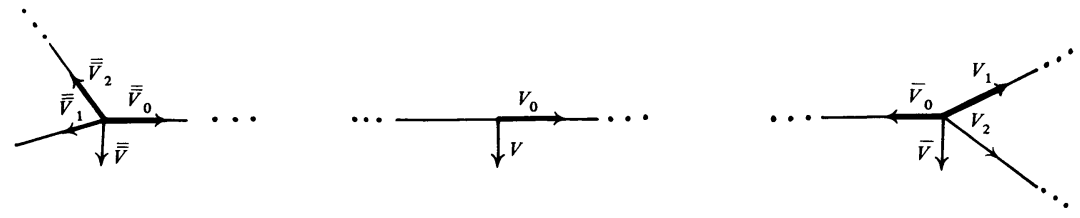

FIG. 5

(This cannot happen unless $\overline{\bar{V}}_{1}$ and $\overline{\bar{V}}_{2}$ are interchanged.)

Also the above reasoning shows that the ordering does not depend on which of the parameters was labeled $t_{1}$ and which $t_{2}$ (even though the definition proper does depend on the label).

Lemma 2.5. Let $\gamma \in \Omega^{m}(M)$ be regular and let $x \in \gamma([0,1])-$ $\gamma(\{0,1 / m, \cdots, 1\})$. Let $V$ be a unit vector at $x$ perpendicular to $\gamma$. Then the ordering on the finite set $\gamma^{-1}(x)$ has the following properties:

(a) For $t_{1}, t_{2} \in \gamma^{-1}(x)$ either $t_{1}>t_{2}, t_{2} \underset{V}{>} t_{1}$, or $t_{2}=t_{1}$.

(b) If $t_{1}>t_{2}$, then $t_{2} \rightarrow t_{1}$.

(c) If $t_{1}>t_{2}$ and $\gamma\left(t\left(S_{t_{1}}+S\right)\right)=\gamma\left(t\left(S_{t_{2}} \pm S\right)\right)$ for all $S$ in some interval about 0 , then $t\left(S_{t_{1}}+S\right) \underset{\bar{V}}{>} t\left(S_{t_{2}} \pm S\right)$ for all $S$ in that interval where $\bar{V}$ is the unit vector at $\gamma\left(t\left(S_{t_{1}}+S\right)\right)$ perpendicular to $\gamma$ and giving the same orientation which $V$ gave.

(d) If $t_{1}>t_{2}$ and $t_{2}>t_{3}$, then $t_{1}>t_{3}$.

Proof. Properties (a), (b), and (c) follow directly from the definition. Property (d) also follows from the definition by checking the three cases:

(i) $S_{1}\left(t_{1}, t_{2}\right)<S_{1}\left(t_{2}, t_{3}\right)$,

(ii) $S_{1}\left(t_{1}, t_{2}\right)>S_{1}\left(t_{2}, t_{3}\right)$,

(iii) $S_{1}\left(t_{1}, t_{2}\right)=S_{1}\left(t_{1}, t_{2}\right)$. 
For case (i) we see that $S_{1}\left(t_{1}, t_{3}\right)=S_{1}\left(t_{1}, t_{2}\right)$, and the measured angles corresponding to $t_{1}$ and $t_{3}$ are the same as those for $t_{1}$ and $t_{2}$ respectively. Hence $t_{1}>t_{3}$. The others are similar.

Let $K$ be a connected component of $M-\gamma[0,1]$, and $(x, V)$ be such that $x \in \partial K-\gamma(\{(i / m) \mid i=1, \cdots, m\})$ and $V$ is an inwardly pointing (towards $K$ ) unit vector perpendicular to $\gamma$ at $x$. (Note for given $x$ it could happen that both $V$ and $-V$ point inward toward $K$.) Then the boundary parameter for $K$ at $(x, V)$ is that element of $\gamma^{-1}(x)$ which is maximal with respect to $>$.

If $K$ is a component of $M-\gamma$, and an open interval $(a, b)$ are boundary parameters for $K$, then we define $a$ and $b$ to be boundary parameters for $K$ at $\gamma(a)$ and $\gamma(b)$ respectively. This is to take care of the case $x \in \gamma(\{i / m \mid i=$ $1,2, \cdots, m\})$. One should note that for such an $x$ there may be many boundary parameters of $K$ in $\gamma^{-1}(x)$.

Lemma 2.6. Let $K$ be a connected component of $M-\gamma$. If $K \subset M^{+}(\gamma)$ (where $\gamma$ is regular) and $[a, b]$ is a boundary parameter interval for $K$, then the orientation of $\gamma_{[[a, b]}$ agrees with the orientation of the boundary of $K$. If $K \subset M^{-}$ $(\gamma)$, then the opposite is true. In particular, if $i / m \in(a, b)$ and $i$ is $a+$ vertex (resp. - vertex) and $K \subset M^{+}(\gamma)$ (resp. $M^{-}(\gamma)$ ), then the angle at $i / m$ is convex to $K$.

Proof. See appendix.

Let $\gamma$ be a regular element of $\Omega^{m}(M)$, and $i$ a + vertex. Then $i$ is called a free + vertex if $\left[\frac{i-1}{m}, \frac{i+1}{m}\right]$ is a boundary parameter interval for a component $K \subset M^{+}(\gamma)$. Similarly define a free-vertex.

If $i$ is a + vertex of $\gamma$ and for some $\varepsilon>0,\left[\frac{i}{m}-\varepsilon, \frac{i+1}{m}\right]$ or $\left[\frac{i-1}{m}, \frac{i}{m}+\varepsilon\right]$ is a boundary parameter interval for a component $K \subset M^{+}(\gamma)$, then $i$ is called a half free + vertex. Similarly define a half free-vertex.

A component $K$ of $M-\gamma$ is called a cul-de-sac if the set of boundary parameters of $K$ consists of a single interval $[a, b]$ (hence $\partial K=\gamma([a, b])$ ).

Lemma 2.7. Given a regular $\gamma \in \Omega^{m}(M)$, then either $M^{+}(\gamma)$ is connected or there are at least two cul-de-sacs $K_{1}$ and $K_{2}$ in $\mathrm{M}^{+}(\gamma)$. Similarly for $\mathrm{M}^{-}(\gamma)$.

Proof. See appendix.

Lemma 2.8. Let $\gamma \in \Omega^{m}(M)$ be regular.

(a) If $M^{+}(\gamma)$ is connected, then all + vertices are free, and $2 \pi=\sum_{i=0}^{m-1} \operatorname{Ext}(i)$ $+\int_{M^{+}(\gamma)} k(x) d x$ (Gauss-Bonnet), where $k$ is the gaussian curvature of $M$.

(b) If $K \subset M^{+}(\gamma)$ is a cul-de-sac with boundary parameter interval $[a, b]$, then $\gamma(a)=\gamma(b)$ and (after reparameterization) $\gamma_{[a, b]} \in \Omega^{\bar{m}}(M)$ for some $\bar{m}$, $\gamma_{\mid[a, b]}$ is regular, and $M^{+}\left(\gamma_{\mid[a, b]}\right)=K$. Further one of $a$ or $b$ is a vertex of $\gamma$, say $a=i / m$. Let $j$ be the largest integer such that $j / m \in[a, b]$. If $i<k<j$, and $k$ is $a+$ vertex, then $k$ is free. If $j / m \neq b$, and $j$ is $a+$ vertex, then $j$ is half free. 
If we let $A$ represents the exterior angle for $\gamma_{[a, b]}$ at $\gamma(a)=\gamma(b)$, then $-\pi<A$ $<\pi$ and (assuming $j / m \neq b$ ) $2 \pi=\sum_{k=i+1}^{j} \operatorname{Ext}(k)+A+\int_{K} k(x) d x$, where $k(x)$ is the gaussian curvature of $M$.

Proof. See appendix.

Lemma 2.9. Let $\gamma \in \Omega^{m}(M)$ be regular, and $A \geqslant 0$ a fixed number. Let $i$ be $a$ half free + vertex $($ or - vertex $)$ such that $l_{i}<c, l_{i+1}<c$, and $\operatorname{Ext}(i)>A$. Then for small $\varepsilon>0$ define the closed piecewise geodesic curve $\bar{\gamma}_{\varepsilon}$ by $\bar{\gamma}_{\varepsilon}(\mathrm{j} / \mathrm{m})=$ $\gamma(j / m)$ for $j \neq i$ and $\bar{\gamma}_{\varepsilon}(i / m)=\gamma\left(t\left(S_{i / m} \pm \varepsilon\right)\right)$, where the + is determined by whether $\left[\frac{i-1}{m}, \frac{i}{m}+\varepsilon\right]$ or $\left[\frac{i}{m}-\varepsilon, \frac{i+1}{m}\right]$ is the boundary parameter of a component. (This is the deformation shown in Fig. 3.) Then for sufficiently small $\varepsilon, \bar{\gamma}_{\varepsilon}$ satisfies:

(a) $\bar{\gamma}_{\varepsilon} \in \Omega^{m}(M)$,

(b) $L\left(\bar{\gamma}_{\varepsilon}\right)<L(\gamma)$

(c) $\operatorname{Ext}(\bar{i})>A$, and $i$ is a half free + vertex.

Proof. See appendix.

Lemma 2.10. Let $\gamma \in \Omega^{m}(M)$ be regular, and $i$ be a free + vertex (or vertex) such that $l_{i}<c$ and $l_{i+1}<c$. (The condition $l_{i}<c$ and $l_{i+1}<c$ is not needed.) Define $\bar{\gamma}_{\varepsilon}$ to be the piecewise geodesic with $\bar{\gamma}_{\varepsilon}(j / m)=\gamma(j / m)$ for $j \neq i$ and $\bar{\gamma}_{\varepsilon}(i / m)=\tau(\varepsilon)$ where $\tau$ is the unit speed geodesic emanating from $\gamma(i / m)$ such that $\left\langle V_{1}, \tau^{\prime}(0)\right\rangle=\left\langle V_{2}, \tau^{\prime}(0)\right\rangle\left\langle\pi / 2, V_{1}\right.$ and $V_{2}$ being unit tangent vectors at $\gamma(i / m)$ tangent to $-\gamma_{\left[\frac{i-1}{m}, \frac{i}{m}\right]}$ and $\gamma_{\left[\frac{i}{m}, \frac{i+1}{m}\right]}$ respectively. (This is the deformation in Fig. 1.) Then for sufficiently small $\varepsilon, \bar{\gamma}_{\varepsilon}$ satisfies:

(a) $\bar{\gamma}_{\varepsilon} \in \Omega^{m}(M)$,

(b) $L\left(\bar{\gamma}_{\varepsilon}\right)<L(\gamma)$

(c) $E\left(\bar{\gamma}_{\varepsilon}\right)<E(\gamma)$.

Proof. $\quad \bar{\gamma}_{\varepsilon}$ can be achieved by making two deformations as in Lemma 1.9 (with $A=0$ ) hence $\bar{\gamma}_{\varepsilon} \in \Omega^{m}(M)$. The first variation formula shows that $\bar{l}_{i}<l_{i}$ and $\overline{l_{i+1}}<l_{i+1}$, hence both $b$ and $c$ follow for small $\varepsilon>0$.

Definition. $\Omega_{1 / 2}^{m}(M)=\left\{\gamma \in \Omega^{m}(M) \mid \gamma\right.$ is nondegenerate, and $\int_{M^{+}(\gamma)} K=$ $\left.\int_{M^{-}(\gamma)} K=2 \pi\right\}$ where $K$ is the curvature of $M$.

Lemma 2.11. $\Omega_{1 / 2}^{m}(M)$ is compact and contains the simple closed piecewise geodesics $\gamma$ with $L_{i} \leqslant c$ and such that $\int_{M^{+}(\gamma)} K=\int_{M^{-}(\gamma)} K$.

Proof. See appendix.

Remark. For $\gamma \in \Omega_{1 / 2}^{m}(M)$ both Lemmas 2.2 and 2.3 hold with $\bar{\gamma} \in$ $\Omega_{1 / 2}^{m}(M)$. This follows, since by Lemmas 2.2 (c), 2.3 (c) and 2.4 (d) we see that $\bar{\gamma}$ is nondegenerate, and $M^{+}(\bar{\gamma})-M^{+}(\gamma)$ and $M^{-}(\bar{\gamma})-M^{-}(\gamma)$ are sets of measure 0 (they are subsets of $\gamma[0,1])$. Hence $\int_{M^{+}(\bar{\gamma})} K=\int_{M^{-}(\bar{\gamma})} K$ and $\bar{\gamma} \in$ $\Omega_{1 / 2}^{m}(M)$.

Lemma 2.12. Let $\gamma \in \Omega_{1 / 2}^{m}(M)$ be regular, and $A \geqslant 0$. Assume that there are vertices $i$ and $j$ such that $i$ is a free + vertex, or a half free + vertex with $\operatorname{Ext}(i)>A$, and $j$ is a free - vertex, or a half free - vertex with $|\operatorname{Ext}(j)|>A$. 
Assume further that $l_{i}, l_{i+1}, l_{j}$, and $l_{j+1}$ are all strictly less than $c$. Then by using deformations as in Lemmas 2.9 or 2.10 at the vertices $i$ and $j$ one can construct curves $\gamma_{\varepsilon} \in \Omega_{1 / 2}^{m}(M)$ for sufficiently small $\varepsilon>0$ such that $L\left(\gamma_{\varepsilon}\right)<L(\gamma)$.

Proof. See appendix.

Lemma 2.13. Let $\tau:[0,1] \rightarrow M$ be a simple smooth closed curve such that $\int_{M^{+}(\tau)} K=\int_{M^{-}(\tau)} K$. Then for every $\varepsilon>0$ there is a simple curve $\gamma \in \Omega_{1 / 2}^{m}(M)$ for some $m$ such that $L(\gamma)<L(\tau)+\varepsilon$.

Proof. See appendix.

\section{Proof of the Poincare's problem}

We begin with a result which holds for all compact riemannian manifolds.

Proposition 3.1. Let $M$ be a compact riemannian manifold, and $L>\delta_{0}>0$. Then there is a number $Q\left(M, L, \delta_{0}\right)>0$ depending only on $M, L$ and $\delta_{0}$ such that for all closed piecewise geodesics $\tau$ with $\delta_{0} \leqslant L(\tau) \leqslant L$ and $\sum_{i=1}^{r}\left|\alpha_{i}\right| \leqslant 2 Q$ we have $\left|\alpha_{0}\right|<\pi-Q$, where $\alpha_{i}(i=0,1,2, \cdots, r)$ represents the exterior angle at the ith vertex of $\tau\left(\alpha_{0}\right.$ corresponds to the vertex at $\tau(0)$ and $\tau$ has $r+1$ vertices). Further if $M$ is a convex two-dimensional riemannian manifold, we can find a $Q(M, L)$ (no $\delta_{0}$ dependence) which works for all regular $\tau \in \Omega^{m}(M)$ such that $M^{+}(\tau)$ or $M^{-}(\tau)$ is connected.

Remark. The important part of the above proposition is that $Q$ is independent of the number of vertices of $\tau$. In general the $\delta$ dependence is not important, as for $Q$ small enough there should not exist arbitrarily short closed piecewise geodesics $\tau$ satisfying $\Sigma_{i=1}^{r}\left|\alpha_{i}\right|<Q$. Here we only show this for the case we are interested in.

Proof. Consider the following 4 spaces of curves. In each space the curves $\gamma$ are parameterized on $[0,1]$ proportional to arclength and $L \geqslant L(\gamma) \geqslant \delta_{0}$.

$\Omega(M)=$ space of piecewise geodesics on $M$.

$\Omega(T M)=$ space of broken lines $\gamma$ such that $\gamma \subset T_{p} M$ for some $p \in M$ and $\gamma(0)=0 \in T_{p} M$.

$\Omega_{0}(M)=$ space of geodesic segments in $M$.

$\Omega_{0}(T M)=$ space of line segments $\gamma$ such that $\gamma \subset T_{p} M$ for some $p \in M$ and $\gamma(0)=0 \in T_{p} M$.

Let $U M$ be the unit tangent bundle of $M$ with the usual metric, and let $U T M$ be the unit tangent bundle of $T M$ with the usual metric.

The $c^{1}$ topology on the above spaces is the same as the topology induced by the distance function:

$$
d\left(\gamma_{1}, \gamma_{2}\right)=\max _{t \in[0,1]} d\left(\frac{\gamma_{1}^{\prime}(t)}{\left|\gamma_{1}^{\prime}(t)\right|}, \frac{\gamma_{2}^{\prime}(t)}{\left|\gamma_{2}^{\prime}(t)\right|}\right),
$$


where $d$ on the right-hand side represents the distance in $U M$ or $U T M$ as appropriate. (The fact that we can use the normalized unit vectors follows from the fact that the curves $\gamma_{i}$ are parameterized proportional to arclength and $\delta_{0} \leqslant L\left(\gamma_{i}\right) \leqslant L$ so $\delta_{0} \leqslant\left|\gamma_{i}^{\prime}(t)\right| \leqslant L$.)

Now let $D$ be the development map (see [5] for a definition). We have that $D: \Omega(T M) \rightarrow \Omega(M)$ and $D: \Omega_{0}(T M) \rightarrow \Omega_{0}(M)$ are homeomorphisms (all spaces with the $c^{1}$ topology). We also note that $\Omega_{0}(T M)$ (resp. $\Omega_{0}(M)$ ) is a compact subset of $\Omega(T M)$ (resp. $\Omega(M)$ ). Thus for every $\varepsilon>0$ there is a $\delta(\varepsilon)>0$ such that if $\gamma_{1} \in \Omega(T M)$ and $\gamma_{1} \in \Omega_{0}(T M)$ such that $d_{\Omega(T M)}\left(\gamma_{1}, \gamma_{2}\right)$ $<\delta(\varepsilon)$, then we have $d_{\Omega(M)}\left(D\left(\gamma_{1}\right), D\left(\gamma_{2}\right)\right)<\varepsilon$.

Now let $F: \quad \Omega_{0}(M) \rightarrow \mathbf{R}$ be given by $F(\gamma)=d_{U M}\left(\gamma^{\prime}(0) /\left|\gamma^{\prime}(0)\right|\right.$, $\left.-\gamma^{\prime}(1) /\left|\gamma^{\prime}(1)\right|\right)$. $F$ is continuous on the compact set $\Omega_{0}(M)$ and is never 0 , as no geodesic segment ever has $\gamma^{\prime}(0) /\left|\gamma^{\prime}(0)\right|=-\gamma^{\prime}(1) /\left|\gamma^{\prime}(1)\right|$, hence $F$ has a minimum $q$ on $\Omega_{0}(M)$.

Now choose $Q\left(M, L, \delta_{0}\right)$ small enough such that $2 L \sin (Q)+2 Q<\delta(q / 2)$, $Q<q / 2$ and $Q<\pi / 4$.

Let $\tau$ be as in the theorem, and let $\gamma \in \Omega_{0}(M)$ be the geodesic segment with the same initial tangent vector as $\tau$ and the same length as $\tau$. Let $\bar{\gamma} \in \Omega_{0}(T M)$ and $\bar{\tau} \in \Omega(T M)$ be $D^{-1}(\gamma)$ and $D^{-1}(\tau)$ respectively.

By the definition of $D$ the sum of the absolute values of the exterior angles of $\bar{\tau}$ is the same as that for $\tau$ (except for $\alpha_{0}$ which does not exist in $\bar{\tau}$ as $\bar{\tau}$ is not necessarily closed) in particular the sum is less than or equal to $2 Q$.

Let $\overline{\bar{\tau}}$ be a line segment in $T_{p} M$ (where $p$ is such that $\bar{\gamma}, \bar{\tau} \subset T_{p} M$ ) which makes an angle of $2 Q<\pi / 2$ with $\bar{\gamma}$ (see figure), and has the same length as $\bar{\gamma}$ and $\bar{\tau}$.

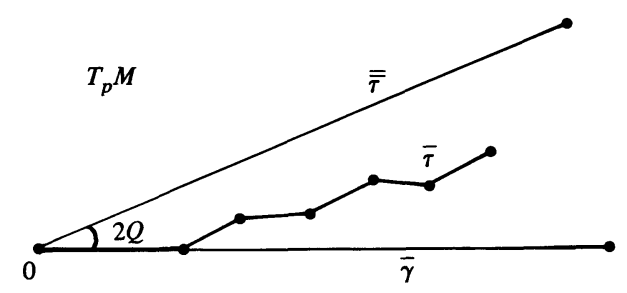

It is a simple exercise in euclidean geometry to show that $d_{\Omega(T M)}(\bar{\tau}, \bar{\gamma}) \leqslant$ $d_{\Omega(T M)}(\overline{\bar{\tau}}, \bar{\gamma})<2 L \sin (Q)+2 Q$. Thus $d_{\Omega(T M)}(\bar{\tau}, \bar{\gamma})<\delta(q / 2)$, and by the definition of $\delta(q / 2)$ we have $d_{\Omega(M)}(\tau, \gamma)<q / 2$. In particular we have $d_{U M}\left(\gamma^{\prime}(1) /\left|\gamma^{\prime}(1)\right|, \tau^{\prime}(1) /\left|\tau^{\prime}(1)\right|\right)<q / 2$. Now using the fact that $\gamma^{\prime}(0)=\tau^{\prime}(0)$ 
we get

$$
\begin{aligned}
d_{U M}\left(\frac{\tau^{\prime}(0)}{\left|\tau^{\prime}(0)\right|},-\frac{\tau^{\prime}(1)}{\left|\tau^{\prime}(1)\right|}\right) \geqslant & d_{U M}\left(\frac{\gamma^{\prime}(0)}{\left|\gamma^{\prime}(0)\right|},-\frac{\gamma^{\prime}(1)}{\left|\gamma^{\prime}(1)\right|}\right) \\
& -d_{U M}\left(-\frac{\gamma^{\prime}(1)}{\left|\gamma^{\prime}(1)\right|},-\frac{\tau(1)}{\left|\tau^{\prime}(1)\right|}\right) \\
& >q-q / 2=q / 2>Q .
\end{aligned}
$$

Thus the absolute value of the interior angle at $\tau(0)$ is greater than $Q$, and hence $\left|\alpha_{0}\right|<\pi-Q$.

To prove the second part of the proposition assume $M$ is convex and two dimensional. Let $\delta_{0}(M)=\sup \left\{r \leqslant c \mid \int_{B_{r}(p)} k \leqslant \pi / 2\right.$ for all $\left.p \in M\right\}$, where $c=\frac{1}{3}$ convexity radius and let $Q(M, L) \equiv Q\left(M, L, \delta_{0}(M)\right)$.

Let $\tau$ be as in the second part of the proposition. If $L(\tau)<\delta_{0}(M) \leqslant c$, then $\tau$ lies inside $B_{\delta_{0}(M) / 2}(\tau(0))$. Hence either $M^{+}(\tau) \subset B_{\delta_{0}(M) / 2}(\tau(0))$ or $M-$ $B_{\delta_{0}(M) / 2}(\tau(0)) \subset M^{+}(\tau)$. Thus by the definition of $\delta_{0}$ and the convexity radius of $M, \int_{M^{+}(\tau)} k>7 \pi / 2$ or $\int_{M^{+}(\tau)} k<\pi / 2$. Now the fact that $\sum_{i=1}^{r}\left|\alpha_{i}\right| \leqslant$ $2 Q(M, L) \leqslant \pi / 2$ and that $\left|\alpha_{0}\right|<\pi$ gives a contradiction to Gauss-Bonnet (Lemma 2.8).

Hence we can assume that $L(\tau) \geqslant \delta_{0}(M)$. In this case the result follows from the first part.

Theorem 3.2. Let $M$ be a convex two-dimensional manifold, and let $\tilde{L}$ be the infimum of the lengths of the smooth simple closed curves on $M$ which split $M$ into two pieces of equal total curvature. Then there is a simple closed geodesic of length $\tilde{L}>0$.

Proof. Let $\bar{L}=\inf \left\{L(\gamma) \mid \gamma \in \Omega_{1 / 2}^{m}(M)\right.$ for some $\left.m\right\}$. By Lemma 2.13 we see that $\tilde{L} \geqslant \bar{L}$. By Corollary $1.4 \bar{L} \geqslant 4 r_{0}>0$ where $r_{0}$ is the radius of the largest ball enclosed by $M$ (when $M$ is isometrically embedded in $\mathbf{R}^{3}$ ). In particular if we find a simple closed geodesic $\gamma$ of length $\bar{L}$, then $\gamma$ is nontrivial and, by the Gauss-Bonnet theorem, $\gamma$ splits $M$ into two pieces of equal total curvature, hence $L(\gamma)=\bar{L}=\tilde{L} \geqslant 4 r_{0}>0$ (in fact $2 \pi r_{0}$ by Theorem 1.5).

Let $\varepsilon=1+\cos (\pi-Q(M, 2 \bar{L}))$ where $Q(M, 2 \bar{L})$ (henceforth referred to as $Q)$ is defined as in Proposition 3.1.

Choose $L_{0}$ such that

(i) $2 \bar{L}>L_{0}>\bar{L}$, and

(ii) $\left(1+L_{0}^{2}\right)\left(L_{0}-\bar{L}\right)<\frac{1}{2} \varepsilon c$, where $c=\frac{1}{3}$ convexity radius.

Let $\gamma_{0} \in \Omega_{1 / 2}^{m}(M)$ for some $m$ be such that $L\left(\gamma_{0}\right)<L_{0}$. By splitting each segment of $\gamma_{0}$ into more pieces, we may assume

(iii) $m>E\left(\gamma_{0}\right)\left(1+L_{0}^{2}\right) / c^{2} L_{0}^{2}$.

Let $\Omega$ be $\Omega_{1 / 2}^{m}(M) \cap\left\{\gamma \mid L(\gamma) \leqslant L\left(\gamma_{0}\right)\right\}$. $\Omega$ is compact (since $\Omega_{1 / 2}^{m}(M)$ is compact by Lemma 2.11) and nonempty as $\gamma_{0} \in \Omega$. 
Let $F: \Omega \rightarrow \mathbf{R}$ be the functional $F(\gamma)=\frac{1}{m c} E(\gamma)+\frac{2}{\varepsilon} L(\gamma)$. Let $\tau$ be a minimum point of $F$ on $\Omega$. By the definition of $\Omega, L(\tau) \leqslant L\left(\gamma_{0}\right)$. We will now show that $\tau$ is a simple closed geodesic of length $\bar{L}$.

Let $l_{i}=L\left(\tau_{\left[\frac{i-1}{m}, \frac{i}{m}\right]}\right)$. We claim that $l_{i}<c$ for all $i$.

To see this we note that $F(\tau) \leqslant F\left(\gamma_{0}\right)$, so

$$
\frac{1}{m c} E(\tau)+\frac{2}{\varepsilon} L(\tau) \leqslant \frac{1}{m c} E\left(\gamma_{0}\right)+\frac{2}{\varepsilon} L\left(\gamma_{0}\right) \text {. }
$$

Hence

$$
E(\tau) \leqslant E\left(\gamma_{0}\right)+\frac{2 m c}{\varepsilon}\left[L_{0}-L(\tau)\right] \leqslant E\left(\gamma_{0}\right)+\frac{2 m c}{\varepsilon}\left[L_{0}-\bar{L}\right],
$$

so by (i) and (ii)

$$
E(\tau)<m c^{2} L_{0}^{2} /\left(1+L_{0}^{2}\right)+m c^{2} /\left(1+L_{0}^{2}\right)=m c^{2} .
$$

Thus we have $m \sum_{i=1}^{m} l_{i}^{2}=E(\tau)<m c^{2}$ and so for each $i, l_{i}<c$ and the claim is shown.

We next note that $\tau$ is regular. For if not, by the remark following Lemma 2.11 and by Lemmas 2.2 and 2.3 , there would be a $\tilde{\tau} \in \Omega_{1 / 2}^{m}(M)$ with $L(\bar{\tau}) \leqslant L(\tau)$ and $E(\bar{\tau})<E(\tau)$. But then $\bar{\tau} \in \Omega$ and $F(\bar{\tau})<F(\tau)$ which contradicts the definition of $\tau$.

We now claim that for either + or - , without loss of generality we will say + (if need be reverse the orientation of $\gamma$ ), there are no free + vertices and no half free + vertices with exterior angle greater than $Q$.

To see this, assume it is not true. In which case there are vertics $i$ and $j$ such that $i$ (resp. $j)$ is a free + (resp. - ) vertex or a half free + (resp. -$)$ vertex with exterior angle greater than $Q$ (resp. less than $-Q$ ).

By Lemma 2.12 and the fact that $l_{i}<c$ for small $\delta$ there is a $\tau_{\delta} \in \Omega_{1 / 2}^{m}(M)$ such that $L\left(\tau_{\delta}\right)<L(\tau)$. Thus $\tau_{\delta} \in \Omega$ for each $\delta$. If both $i$ and $j$ were free, we also know (Lemma 2.10) that $E\left(\tau_{\delta}\right)<E(\tau)$ which contradicts the definition of $\tau$. If one or both of $i$ and $j$ are half free, then $E\left(\tau_{\delta}\right)$ could be larger than $E(\tau)$. To get a contradiction we must show $F\left(\tau_{\delta}\right)<F(\tau)$. Since $\tau_{\delta}$ is built out of deformations described in Lemma 2.9, it is enough to show that these deformations are $F$ decreasing. To do this we compute $F^{\prime}(0)$ for this deformation applied to vertex $i$.

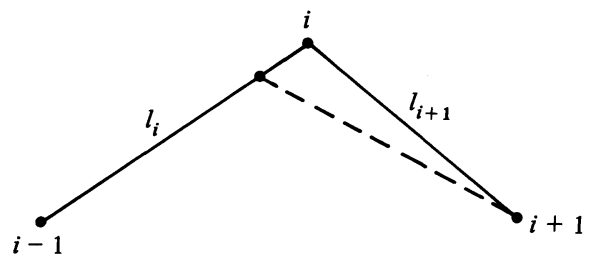


$L^{\prime}(0)=l_{i}^{\prime}+l_{i+1}^{\prime}=-1+l_{i+1}^{\prime}<-1+(1-\varepsilon)=-\varepsilon$ where the inequality follows from the first variation formula, the fact that the exterior angle is greater than $Q$, and the definition of $\varepsilon$. Since

$$
E^{\prime}(0)=m\left(2 l_{i} l_{i}^{\prime}+2 l_{i+1} l_{i+1}^{\prime}\right) \leqslant 2 m l_{i+1} l_{i+1}^{\prime}<2 m c l_{i+1}^{\prime}<2 m c(1-\varepsilon)<2 m c,
$$

we have

$$
F^{\prime}(0)=\frac{1}{m c} E^{\prime}(0)+\frac{2}{\varepsilon} L^{\prime}(0)<2-2=0 .
$$

The above argument shows that the deformation is $F$ decreasing as long as $l_{i+1}<c$, and the exterior angle stays less than $Q$. Since $\tau_{\delta}$ is constructed from such deformations, where $l_{i+1}$ stays less than $c$ and the exterior angle stays larger than $Q$, we see that $F\left(\tau_{\delta}\right)<F(\tau)$. This contradicts the definition of $\tau$, and the claim follows.

We now show that $M^{+}(\tau)$ is connected. Assume not, then by Lemma 2.7 there are at least two cul-de-sacs $K_{1}, K_{2} \subset M^{+}(\tau)$. Hence for at least one of these, say $K_{1}$, we have $\int_{K_{1}} K \leqslant \pi$.

Since $K_{1}$ is a cul-de-sac, $\partial K_{1}=\tau[a, b]$ where $[a, b]$ are the boundary parameter values for $K_{1}$. By Lemma 2.8 we may assume that $a=i / m$, and we let $j$ be the largest integer such that $j / m \leqslant b$. For $i<k<j$ we have $\operatorname{Ext}(k) \leqslant 0$, since there are no free + vertices and by Lemma 2.8 any such $k$ which is a + vertex must be free. Also by Lemma 2.8 and the previous claim, if $j / m \neq b$ then $\operatorname{Ext}(j) \leqslant Q$. Let $A$ be the exterior angle of $\tau_{\mid[a, b]}$ at $\tau(a)=\tau(b) . A<\pi$ by Lemma 2.8 .

We consider two cases. First assume that $b=j / m$ (i.e., $b$ is a vertex of $\tau$ ). In this case Gauss-Bonnet (Lemma 2.8) gives $2 \pi=\sum_{k=i+1}^{j-1} \operatorname{Ext}(k)+A+\int_{K_{1}} K<$ $0+\pi+\pi=2 \pi$. This gives a contradiction. Now we assume that $b \neq j / m$. In this case Gauss-Bonnet gives (Lemma 2.8)

(iv) $2 \pi=\sum_{k=i+1}^{j-1} \operatorname{Ext}(k)+\operatorname{Ext}(j)+A+\int_{K_{1}} k<\sum_{k=i+1}^{j-1} \operatorname{Ext}(k)+Q+2 \pi$.

So $\sum_{k=i+1}^{j-1} \operatorname{Ext}(k)>-Q$. Since $\operatorname{Ext}(k) \leqslant 0$ for each $k$ we see $\sum_{k=i+1}^{j-1}|\operatorname{Ext}(k)|$ $<Q$. Thus $\sum_{k=i+1}^{j}|\operatorname{Ext}(k)|<2 Q$. Thus by Proposition 3.1 we have $A<\pi-Q$. Using this in (iv) we get $2 \pi<0+Q+(\pi-Q)+\pi=2 \pi$, again a contradiction. Hence we have shown that $M^{+}(\tau)$ is connected.

Since by Lemma 2.8 all + vertices are free, and since by a previous claim there are no free + vertices we see there are no + vertices at all. Thus by Gauss-Bonnet (Lemma 2.8) there are no - vertices either and $\tau$ is a geodesic.

The fact that $\tau$ is simple follows from the fact that $\tau$ is the limit of simple curves and hence does not transverse itself more than once nor does it have 
any transverse self intersections, thus it can have no self intersections since it is a geodesic.

To see that $L(\tau)=\bar{L}$, assume not. Let $\bar{L}_{0}$ be such that $\bar{L}<\bar{L}_{0}<L(\tau) \leqslant L_{0}$. The same procedure as above gives rise to a simple closed geodesic $\bar{\tau}$ with $L(\bar{\tau}) \leqslant \bar{L}_{0}<L(\tau)$. But such a $\bar{\tau}$ can be thought of as an element of $\Omega_{1 / 2}^{m}(M)$ (the same $m$ as above), and we have $\bar{\tau} \in \Omega$ since $L(\bar{\tau})<L(\tau) \leqslant L\left(\gamma_{0}\right)$. But under the arclength parameter $F(\bar{\tau})<F(\tau)$, which contradicts the definition. Thus we have $L(\tau)=\bar{L}=\tilde{L}$, and the theorem is proved.

\section{Appendix}

We begin by introducing some notation and preliminary lemmas which will be used throughout the appendix.

Let $\gamma \in \Omega^{m}(M)$ have $l_{i} \neq 0$ for all $i=0,1, \cdots, m-1$. Let $\left\{t_{0}, t_{1}, \cdots, t_{p}\right\}$ be $\gamma^{-1}(\gamma(\{0,1 / m, 2 / m, \cdots,(m-1) / m\}))$ where $0=t_{0}<t_{1}<t_{2}<\cdots<t_{p}$ (of course $p \geqslant m-1)$. Define an equivalence relation $\sim$ on the set $\{0,1, \cdots, p\}$ by $i \sim j$ if $\gamma\left[t_{i}, t_{i+1}\right]=\gamma\left[t_{j}, t_{j+1}\right]$. Let $\left\{I_{1}, \cdots, I_{q}\right\}$ be the partition of $\{0,1, \cdots, p\}$ induced by $\sim$. For each $k \in\{1,2, \cdots, q\}, \gamma\left(I_{k}\right)$ will represent the geodesic segment $\gamma\left[t_{i}, t_{i+1}\right]$ for $i \in I_{k}$.

Define $A(\gamma)=\min \left\{d\left(\gamma(i / m), \gamma\left(I_{k}\right)\right) \mid \gamma(i / m) \notin \gamma\left(I_{k}\right)\right\}$. The only case where $A(\gamma)$ is not defined is when $\gamma$ is a single geodesic segment transversed back and forth. In this case let $A(\gamma)$ be the length of the segment.

Define the normal exponential map $F_{i}:\left[t_{i}, t_{i+1}\right] \times \mathbf{R} \rightarrow M$ for $i \in$ $\{0,1, \cdots, p\}$ by $F_{i}(t, s)=\operatorname{Exp}_{\gamma(t)} s Y_{t}$, where $Y_{t}$ is the unit vector perpendicular to $\gamma^{\prime}(t)$ and such that the orientation given by $\left(\gamma^{\prime}(t), Y_{t}\right)$ is the orientation of $M$.

Let $B(\gamma)=\sup \left\{r\left|F_{i}\right|_{\left(t_{i}, t_{i+1}\right) \times(-r, r)}\right.$ is a diffeomorphism onto its image for all $i=0,1, \cdots, p$. For $r<B(\gamma)$ let $T_{i}(r)=F_{i}\left(\left(t_{i}, t_{i+1}\right) \times(-r, r)\right)$, the normal tube around $\gamma\left(\left(t, t_{i+1}\right)\right)$ of radius $r$.

A number $\varepsilon>0$ is said to be small for $\gamma$ if $\varepsilon<\min \left\{\frac{1}{4} A(\gamma), B(\gamma)\right\}$. For a given small $\varepsilon$ a number $\delta>0$ is said to be much smaller than $\varepsilon$ if for all $i$, $j \in\{0,1, \cdots, p\}$ with $i \nsim j$ we have $T_{i}(\delta) \cap T_{j}(\delta) \subset \cup_{i=0}^{m-1} B_{\varepsilon / 2}(\gamma(i / m))$.

For all small $\varepsilon$ and all $j \in\{0,1, \cdots, p\}$ we define the numbers $a_{j}(\varepsilon)$, $b_{j}(\varepsilon) \in\left(t_{j}, t_{j+1}\right)$ such that $d\left(\gamma\left(t_{j}\right), \gamma\left(a_{j}(\varepsilon)\right)\right)=\varepsilon$ and $d\left(\gamma\left(t_{j+1}\right), \gamma\left(b_{j}(\varepsilon)\right)\right)=\varepsilon$. We note here that $a_{j}(\varepsilon)<b_{j}(\varepsilon)$ since $\varepsilon$ is small.

Lemma A.0. Let $\gamma:[a, b] \rightarrow M$ be a minimizing geodesic segment parameterized by arclength, and $c:[a, b] \rightarrow M$ a piecewise smooth curve such that $d(c(t), \gamma(t))<\delta$. If $t_{0}, t_{1} \in(a, b)$ such that $d\left(\gamma\left(t_{0}\right), c\left(t_{1}\right)\right)<\delta$, then one can reparameterize the curve $c$ (we let $\bar{c}:[a, b] \rightarrow M$ be the curve with the new parameterization) such that $\bar{c}\left(t_{0}\right)=c\left(t_{1}\right)$ and for all $t \in[a, b], d(\gamma(t), \bar{c}(t))<$ $5 \delta$. 
Proof. Assume without loss of generality that $t_{0}<t_{1}$. We see $d\left(\gamma\left(t_{0}\right), \gamma\left(t_{1}\right)\right)<d\left(\gamma\left(t_{0}\right), c\left(t_{1}\right)\right)+d\left(c\left(t_{1}\right), \gamma\left(t_{1}\right)\right)<2 \delta$. Further since $\gamma$ is minimizing, we have $d\left(\gamma\left(t_{0}^{\prime}\right), \gamma(t)\right)<2 \delta$ for $t \in\left[t_{0}, t_{1}\right]$. By the strict inequality there is an $\varepsilon>0$ such that $d\left(\gamma\left(t_{0}\right), \gamma(t)\right)<2 \delta$ for all $t \in\left[t_{0}-\varepsilon, t_{1}+\varepsilon\right]$. We also see that for $t \in\left[t_{0}-\varepsilon, t_{1}+\varepsilon\right], d\left(c(t), \gamma\left(t_{0}\right)\right) \leqslant d(c(t), \gamma(t))+$ $d\left(\gamma(t), \gamma\left(t_{0}\right)\right)<3 \delta$.

Let

$$
\bar{c}(t)= \begin{cases}c(t) & t \notin\left[t_{0}-\varepsilon, t_{1}+\varepsilon\right], \\ c\left(L_{1}(t)\right) & t \in\left[t_{0}-\varepsilon, t_{1}\right], \\ c\left(L_{2}(t)\right) & t \in\left[t_{1}, t_{1}+\varepsilon\right],\end{cases}
$$

where $L_{1}$ is the linear transformation from $\left[t_{0}-\varepsilon, t_{1}\right]$ to $\left[t_{0}-\varepsilon, t_{0}\right]$, and $L_{2}$ is the linear transformation from $\left[t_{1}, t_{1}+\varepsilon\right]$ to $\left[t_{0}, t_{1}+\varepsilon\right]$.

By the definition $\bar{c}\left(t_{1}\right)=c\left(t_{0}\right)$. Let $t \in[a, b]$. If $t \notin\left[t_{0}-\varepsilon, t_{1}+\varepsilon\right]$, then $d(\gamma(t), \bar{c}(t))=d(\gamma(t), c(t))<\delta<5 \delta$. If $t \in\left[t_{0}-\varepsilon, t_{1}+\varepsilon\right]$, then there is a $\bar{t} \in\left[t_{0}-\varepsilon, t_{1}+\varepsilon\right]$ such that $\bar{c}(t)=c(\bar{t})$. Hence

$$
\begin{aligned}
d(\gamma(t), \bar{c}(t)) & =d(\gamma(t), c(\bar{t})) \leqslant d\left(\gamma(t), \gamma\left(t_{0}\right)\right)+d\left(\gamma\left(t_{0}\right), c(\bar{t})\right) \\
& <2 \delta+3 \delta=5 \delta .
\end{aligned}
$$

Lemma A.1. Let $\gamma \in \Omega^{m}(M)$ be such that $l_{i} \neq 0$ for all $i$, and let $\varepsilon>0$ be small for $\gamma$. Then for every $\delta>0$ much smaller than $\varepsilon$ we can find a piecewise smooth approximating curve $c_{\delta}$ as in the definition of $\Omega^{m}(M)$, such that for each $j \in\{0, \cdots, p\}$ there is a $\delta_{j}$ with $-\delta<\delta_{j}<\delta$ such that $c_{\delta}(t)=F_{j}\left(t, \delta_{j}\right)$ for all $t \in\left[a_{j}(\varepsilon), b_{j}(\varepsilon)\right]$.

Proof. Choose $\bar{\delta}<\delta / 5$, and let $c_{\delta}^{-}$be an approximation as in the definition of $\Omega^{m}(M)$.

We will assume for simplicity of argument that $\gamma\left(t_{i}\right)=\gamma\left(t_{j}\right)$ and $\gamma\left(t_{i+1}\right)=$ $\gamma\left(t_{j+1}\right)$ for $i, j \in I^{k}$. (In fact this will not be true if $I^{k}$ consists of more than one element. What we are doing is reversing the orientation of some of the segments. The new approximating curve will also have the wrong orientation on these segments, so to complete the argument one simply reverses the orientation of those segments back to the original orientation.)

For each $j \in\{0, \cdots, p\}$ let $\bar{a}_{j}$ be the number in $\left[t_{j}, a_{j}(\varepsilon)\right]$ such that $d\left(\gamma\left(a_{j}\right), \gamma\left(a_{j}(\varepsilon)\right)\right)=\bar{\delta}$. Similarly define $\bar{b}_{j} \in\left[b_{j}(\varepsilon), t_{j+1}\right]$. Now since $\varepsilon>\delta>5 \bar{\delta}$ we have

(i) $d\left(\gamma\left(\bar{a}_{j}\right), \gamma\left(t_{j}\right)\right)=\varepsilon-\bar{\delta}>4 \bar{\delta}$,

(ii) $d\left(\gamma\left(\bar{a}_{j}\right), \gamma\left(t_{j}\right)\right)=\varepsilon-\bar{\delta}>\varepsilon / 2+\bar{\delta}$,

(iii) $d\left(\gamma\left(b_{j}\right), \gamma\left(t_{j+1}\right)=\varepsilon-\bar{\delta}>4 \bar{\delta}\right.$,

(iv) $d\left(\gamma\left(\bar{b}_{j}\right), \gamma\left(t_{j+1}\right)=\varepsilon-\bar{\delta}>\varepsilon / 2+\bar{\delta}\right.$. 
For each $j \in\{0,1, \cdots, p\}$ let $\tau_{\bar{a}_{j}}$ be the unit speed geodesic $\tau_{\bar{a}_{j}}:[-\bar{\delta}, \bar{\delta}] \rightarrow M$ perpendicular to $\gamma$ at $\bar{a}_{j}\left(\tau_{\bar{a}_{j}}(0) \in \gamma\right)$. Similarly define $\tau_{\bar{b}_{j}}$. We note that if $i \sim j$, then $\tau_{\bar{a}_{i}}=\tau_{\bar{a}_{j}}$ and $\tau_{\overline{b_{i}}}=\tau_{\overline{b_{j}}}$.

Since $c_{\bar{\delta}\left[t_{j}, t_{j+1}\right]}$ is a $\bar{\delta}$ approximation to $\gamma_{\mid\left[t_{j}, t_{j+1}\right]}$, and by (i) above we see that there is a smallest number $t^{\bar{a}_{j}} \in\left(t_{j}, t_{j+1}\right)$ such that $c_{\delta}\left(t^{\bar{a}_{j}}\right) \in \tau_{\bar{a}_{j}}[-\bar{\delta}, \bar{\delta}]$. Similarly there is a largest number $t^{\overline{b_{j}}}$ such that $c_{\bar{\delta}}\left(t^{\overline{b_{j}}}\right) \in \tau_{\overline{b_{j}}}[-\bar{\delta}, \bar{\delta}]$. By applying Lemma A.0 many times (disjointly) we see that there is a reparameterization $\bar{c}$ of $c_{\bar{\delta}}$ which is a $\delta$ approximation and such that $t^{\bar{a}_{j}}=\bar{a}_{j}$ and $t^{\bar{b}_{j}}=\bar{b}_{j}$ for all $j$.

Let $\delta_{j}$ be the number such that $\tau_{\bar{a}_{j}}\left(\delta_{j}\right)=\bar{c}\left(\bar{a}_{j}\right)$, and let $\bar{\delta}_{j}$ be such that $\tau_{b_{j}}\left(\overline{\delta_{j}}\right)=\bar{c}\left(\bar{b}_{j}\right)$. Now define

$$
c_{\delta}(t)= \begin{cases}c(t) & \text { if } t \notin \bigcup_{j=0}^{p}\left[\bar{a}_{j}, \bar{b}_{j}\right], \\ F_{j}\left(t, \delta_{j}\right) & \text { if } t \in\left[\bar{a}_{j}, b_{j}\right], \\ F_{j}\left(t, L_{j}(t)\right) & \text { if } t \in\left[b_{j}, \bar{b}_{j}\right],\end{cases}
$$

where $L_{j}(t)$ is the linear transformation from $\left[b_{j}, \bar{b}_{j}\right]$ to $\left[\delta_{j}, \bar{\delta}_{j}\right]$.

It is clear that $c_{\delta}(t)$ is piecewise smooth and a $\delta$-approximation to $\gamma$ (since $\delta_{j}<\bar{\delta}<\delta$ and $\bar{\delta}_{j}<\bar{\delta}<\delta$ ) so we need only show that $c_{\delta}$ is simple. Assume $c_{\delta}\left(t_{0}\right)=c_{\delta}\left(t_{1}\right)$. We will show $t_{0}=t_{1}$ by considering all the cases.

We first note that for $i \nsim j$

$$
\left[T_{i}(\bar{\delta}) \cup \bigcup_{k=0}^{m-1} B_{\varepsilon / 2}\left(\gamma\left(\frac{k}{m}\right)\right)\right] \cap F_{j}\left(\left[\bar{a}_{j}, \bar{b}_{j}\right] \times[-\bar{\delta}, \bar{\delta}]\right)=\varnothing .
$$

This follows from the fact that $\bar{\delta}$ is much smaller than $\varepsilon$ and properties (ii) and (iv). Now if $t \in\left[t_{i}, t_{i+1}\right]$, then $c_{\delta}(t) \in\left[T_{i}(\bar{\delta}) \cup \cup_{k=0}^{m-1} B_{\varepsilon / 2}(\gamma(i / m))\right]$. This follows since $c_{\delta}(t)$ is $\bar{\delta}$ close to $\gamma(\bar{t})$ for some $\bar{t} \in\left[t_{i}, t_{i+1}\right]$ ( $\bar{t}$ need not be $t$ since we reparameterized the original approximation).

Thus by the above and property (v) we may assume that $t_{0} \in\left[t_{i}, t_{i+1}\right]$ and $t_{1} \in\left[t_{j}, t_{j+1}\right]$ where $i \sim j$. Since $i \sim j$ by the definitions, we have $\gamma\left(\bar{a}_{i}\right)=\gamma\left(\bar{a}_{j}\right)$, $\gamma\left(a_{i}\right)=\gamma\left(a_{j}\right), \gamma\left(b_{i}\right)=\gamma\left(b_{j}\right), \gamma\left(\bar{b}_{i}\right)=\gamma\left(\bar{b}_{j}\right), \tau_{\bar{a}_{i}}=\tau_{\bar{a}_{j}}$, and $\tau_{\bar{b}_{i}}=\tau_{\bar{b}_{j}}$. Since $c$ was simple we have $\delta_{i} \neq \delta_{j}$ and $\bar{\delta}_{i} \neq \bar{\delta}_{j}$. Also since $c$ had no self intersection, if $\delta_{i}<\delta_{j}$ then $\bar{\delta}_{i}<\bar{\delta}_{j}$. From this it is easy to see that either $t_{0} \notin\left[\bar{a}_{i}, \bar{b}_{i}\right]$ or $t_{1} \notin\left[\bar{a}_{j}, \bar{b}_{j}\right]$, or $t_{0}=t_{1}$.

Now assume $t_{0} \notin\left[\bar{a}_{i}, \bar{b}_{i}\right]$. Then by the definition of $c\left(\bar{a}_{i}\right)$ (that is the reparameterization of $\left.c_{\bar{\delta}}\right) c_{\delta}\left(t_{0}\right) \notin F_{i}\left(\left[\bar{a}_{i}, \bar{b}_{i}\right] \times[-\delta, \delta]\right)=F_{j}\left(\left[\bar{a}_{j}, \bar{b}_{j}\right] \times\right.$ $[-\delta, \delta])$, hence $t_{1} \notin\left[\bar{a}_{j}, \bar{b}_{j}\right]$. Thus we may assume that $t_{0}, t_{1} \notin \cup_{k=0}^{p}\left[\bar{a}_{k}, \bar{b}_{k}\right]$, but then $c_{\delta}\left(t_{0}\right)=c\left(t_{0}\right)$ and $c_{\delta}\left(t_{1}\right)=c\left(t_{1}\right)$ so that $t_{0}=t_{1}$ since $c$ is simple.

We now prove the lemmas of $\$ 2$. 
Proof of Lemma 2.2. By the definition of $\bar{\gamma}$ it is clear that it satisfies the conclusions (b) and (c) of the lemma. It is also clear that to show $\bar{\gamma} \in \Omega^{m}(M)$ we need only show that $\bar{\gamma}$ is the limit of simple curves.

Fix $\varepsilon>0$, and let $c_{\varepsilon / 2}$ be an approximation to $\gamma$ as in the definition of $\Omega^{m}(M)$. Choose $a \in\left[\frac{i-1}{m}, \frac{i}{m}\right]$ so small that $d\left(\gamma\left(\frac{i}{m}\right), \gamma\left(\frac{1}{2}\left(a-\frac{i-1}{m}\right)+\frac{i}{m}\right)\right)<\varepsilon / 2$. It is clear that $d\left(\gamma\left(t_{0}\right), \gamma\left(t_{1}\right)\right)<\varepsilon / 2$ for $t_{0}, t_{1} \in\left[\frac{i-1}{m}, \frac{1}{2}\left(a-\frac{i-1}{m}\right)+\frac{i}{m}\right]$.

Define $\bar{c}_{\varepsilon}(t)$ by

$$
\bar{c}_{\varepsilon}(t)= \begin{cases}c_{\varepsilon / 2}(t) & t \notin\left[\frac{i-1}{m}, \frac{i+1}{m}\right], \\ c_{\varepsilon / 2}(L(t)) & t \in\left[\frac{i-1}{m}, a\right], \\ c_{\varepsilon / 2}\left(\frac{1}{2}\left(t-\frac{i-1}{m}\right)+\frac{i}{m}\right) & t \in\left[a, \frac{i+1}{m}\right],\end{cases}
$$

where $L$ is the linear transformation taking $\left[\frac{i-1}{m}, a\right]$ to $\left[\frac{i-1}{m}, \frac{1}{2}\left(a-\frac{i-1}{m}\right)+\frac{i}{m}\right]$. Since $\bar{c}_{\varepsilon}(t)$ is just a reparameterization of $c_{\varepsilon / 2}$, it is simple. Thus we only need to show that $d\left(\bar{\gamma}(t), \bar{c}_{\varepsilon}(t)\right)<\varepsilon$ for $t \in[0,1]$.

For $t \notin\left[\frac{i-1}{m}, \frac{i+1}{m}\right]$

$$
d\left(\bar{\gamma}(t), \bar{c}_{\varepsilon}(t)\right)=d\left(\gamma(t), c_{\varepsilon / 2}(t)\right)<\varepsilon / 2<\varepsilon .
$$

For $t \in\left[a, \frac{i+1}{m}\right]$

$$
\begin{aligned}
d\left(\bar{\gamma}(t), \bar{c}_{\varepsilon}(t)\right) & =d\left(\gamma\left(\frac{1}{2}\left(t-\frac{i-1}{m}\right)+\frac{i}{m}\right), c_{\varepsilon / 2}\left(\frac{1}{2}\left(t-\frac{i-1}{m}\right)+\frac{i}{m}\right)\right) \\
& <\varepsilon / 2<\varepsilon .
\end{aligned}
$$

For $t \in\left[\frac{i-1}{m}, a\right]$

$$
\begin{aligned}
d\left(\bar{\gamma}(t), \bar{c}_{\varepsilon}(t)\right)= & d\left(\gamma\left(\frac{1}{2}\left(t-\frac{i-1}{m}\right)+\frac{i}{m}\right), c_{\varepsilon / 2}(L(t))\right) \\
\leqslant & d\left(\gamma\left(\frac{1}{2}\left(t-\frac{i-1}{m}\right)+\frac{i}{m}\right), \gamma(L(t))\right) \\
& +d\left(\gamma(L(t)), c_{\varepsilon / 2}(L(t))\right) \\
< & \varepsilon / 2+\varepsilon / 2=\varepsilon .
\end{aligned}
$$

Proof of Lemma 2.3. Let $t_{i_{0}}=i / \mathrm{m}$. Since $\gamma$ makes a $180^{\circ}$ turn at $i / m$, $\gamma\left(\left[t_{i_{0}-1}, t_{i_{0}}\right]\right)=\gamma\left(\left[t_{i_{0}}, t_{i_{0}+1}\right]\right)$, i.e., $i_{0}-1 \sim i_{0}$. Let $I \in\left\{I^{1}, I^{2}, \cdots, I^{q}\right\}$ be the set containing $i_{0}$, i.e., $j \in I$ if $j \sim i_{0}$. Let $I_{0} \subset I$ be $\left\{j \in I \mid \gamma\left(t_{j}\right)=\gamma\left(t_{i_{0}}\right)\right.$, $\gamma\left(t_{j+1}\right)=\gamma\left(t_{i_{0}+1}\right)$ and $\left.\gamma\left(t_{j-1}\right)=\gamma\left(t_{i_{0}-1}\right)\right\}$, i.e., if $j \in I_{0}$, then $\gamma$ make a $180^{\circ}$ turn at $t_{j}$, in particular $t_{j}=k / m$ for some $k \in\{0,1, \cdots, m-1\}$.

Fix $\varepsilon>0$ small relative to $\gamma$. For all integers $N$ such that $1 / N$ is much smaller than $\varepsilon$ (see definitions at the beginning of the appendix for the 
definition of "small" and "much smaller"), let $C_{1 / N}$ be a simple piecewise smooth approximation to $\gamma$ as in Lemma A.1.

Let $Y$ be a unit vector perpendicular to $\gamma$ at $\gamma\left(\frac{1}{2}\left(t_{i_{0}}+t_{i_{0}+1}\right)\right.$ ) (same as $\gamma\left(\frac{1}{2}\left(t_{j}+t_{j+1}\right)\right)$ for $\left.j \in I\right)$. Then by Lemma A.1 for each $j \in I$ there is a $\delta_{j}^{N}$ such that $C_{1 / N}\left(\frac{1}{2}\left(t_{j}+t_{j+1}\right)\right)=\sigma\left(\delta_{j}^{N}\right)$, where $\sigma$ is the unit speed geodesic with $\sigma^{\prime}(0)=Y$, and $-1 / N<\delta_{j}^{N}<1 / N$. Further $\delta_{j}^{N}=\delta_{k}^{N}$ implies $j=k$.

Choose $j_{N} \in I_{0}$ such that for all $j \in I_{0}$ we have $\left|\delta_{j_{N}-1}^{N}-\delta_{j_{N}}^{N}\right| \leqslant\left|\delta_{j-1}^{N}-\delta_{j}^{N}\right|$.

We claim that for all $i \in I, \delta_{i}^{N} \notin\left(\delta_{j_{N}-1}^{N}, \delta_{j_{N}}^{N}\right)$. To see this let $\tau$ be the simple closed curve constructed by joining

$$
C_{1 / N}\left[\frac{1}{2}\left(t_{j_{N}-1}+t_{j_{N}}\right), \frac{1}{2}\left(t_{j_{N}+1}+t_{j_{N}}\right)\right] \text { to } \sigma\left(\left[\delta_{j_{N}-1}^{N}, \delta_{j_{N}}^{N}\right]\right) \text {. }
$$

$\tau$ splits $M$ into two disjoint pieces since it is a simple closed curve. Now assume that $\delta_{i}^{N} \in\left(\delta_{j_{N}-1}^{N}, \delta_{j_{N}}^{N}\right)$, and further without loss of generality that $\gamma\left(t_{i}\right)=$ $\gamma\left(t_{j_{N}-1}\right)$ and $\gamma\left(t_{i+1}\right)=\gamma\left(t_{j_{N}}\right)$. (If not, reverse the orientation in the following argument.) By the definition of $C_{1 / N}$ (see Lemma A.1) and the fact that $\delta_{i}^{N} \in\left(\delta_{j_{N}-1}^{N}, \delta_{j_{N}}^{N}\right)$ we see that for small $\bar{\varepsilon}>0, C_{1 / N}\left(\frac{1}{2}\left(t_{i}+t_{i+1}\right)+\bar{\varepsilon}\right)$ is inside $\tau$, while $C_{1 / N}\left(t_{i}\right)$ and $C_{1 / N}\left(t_{i+2}\right)$ are outside since they are both near vertices of $\gamma$ which are not $\gamma(i / m))$. Thus $c_{1 / N \mid\left[t_{i}, t_{i+2}\right]}$ must intersect $\tau$ again. Since $c_{1 / N}$ is simple, it must in fact intersect $\left.\sigma\right|_{\left(\delta_{j_{N}-1}^{N}, \delta_{j N}^{N}\right)}$ again. This point of intersection must be $c_{1 / N}\left(\frac{1}{2}\left(t_{i+1}+t_{i+2}\right)\right.$ by the definition of $c_{1 / N}$ (Lemma A.1) again. This implies that $i+1 \in I_{0}$ and $\delta_{i+1}^{N}, \delta_{i}^{N} \in\left(\delta_{j_{N}-1}^{N}, \delta_{j_{N}}^{N}\right)$ contradicting the minimality of $j_{N}$. Thus we have shown the claim.

Since $I_{0}$ is a finite set, there is a $\bar{j} \in I_{0}$ such that $\bar{j}=j_{N}$ for an infinite number of $N$. Let $k$ be such that $k / m=t_{j}$ (such a $k$ exists since $\left.j \in I_{0}\right)$. We thus have $\left[t_{j-1}, t_{j+1}\right] \subset\left[\frac{k-1}{m}, \frac{k+1}{m}\right]$.

Now we can define

$$
\gamma(t)= \begin{cases}\gamma(t) & \text { if } t \notin\left[\frac{k-1}{m}, \frac{k+1}{m}\right], \\ \gamma\left(L_{k-1}(t)\right) & \text { if } t \in\left[\frac{k-1}{m}, \frac{k}{m}\right], \\ \gamma\left(L_{k}(t)\right) & \text { if } t \in\left[\frac{k}{m}, \frac{k+1}{m}\right],\end{cases}
$$

where $L_{k-1}$ is the linear transformation from $\left[\frac{k-1}{m}, \frac{k}{m}\right]$ to $\left[\frac{k-1}{m}, \frac{1}{2}\left(t_{j-1}+t_{j}\right)\right]$, and $L_{k}$ is the linear transformation from $\left[\frac{k}{m}, \frac{k+1}{m}\right]$ to $\left[\frac{1}{2}\left(t_{j}+t_{j+1}\right), \frac{k+1}{m}\right]$.

It is clear that in order to complete the proof of the lemma the only thing to verify is that $\bar{\gamma}$ is the limit of simple curves. 
Let $\bar{\varepsilon}>0$. Choose $N$ so that $1 / N$ is much smaller than $\varepsilon, 1 / N<\bar{\varepsilon}$, and $j_{N}=\bar{j}$. Now define

$$
\bar{c}_{\bar{\varepsilon}}(t)= \begin{cases}c_{1 / N}(t) & t \notin\left[\frac{k-1}{m}, \frac{k+1}{m}\right], \\ c_{1 / N}\left(L_{k-1}(t)\right) & t \in\left[\frac{k-1}{m}, \frac{k}{m}-\delta_{1}\right], \\ F_{j-1}\left(L_{k-1}(t), L_{1}(t)\right) & t \in\left[\frac{k}{m}-\delta_{1}, \frac{k}{m}\right], \\ F_{j}\left(L_{k}(t), L_{2}(t)\right) & t \in\left[\frac{k}{m}, \frac{k}{m}+\delta_{2}\right], \\ c_{1 / N}\left(L_{k}(t)\right) & t \in\left[\frac{k}{m}+\delta_{2}, \frac{k+1}{m}\right],\end{cases}
$$

where $L_{k}$ and $L_{k-1}$ are as above, $L_{1}$ is the linear transformation from $\left[\frac{k}{m}-\delta_{1}, \frac{k}{m}\right]$ to $\left[\delta_{j-1}^{N}, 0\right]$, and $L_{2}$ is the linear transformation from $\left[\frac{k}{m}, \frac{k}{m}+\delta_{2}\right]$ to $\left[0, \delta \frac{N}{j}\right] . \delta_{1}$ and $\delta_{2}$ are chosen so small that $d\left(\gamma\left(L_{k-1}\left(\frac{k}{m}-\delta_{1}\right)\right), \gamma\left(L_{k-1}\left(\frac{k}{m}\right)\right)\right)<1 / N$ and $d\left(\gamma\left(L_{k}\left(\frac{k}{m}+\delta_{2}\right)\right), \gamma\left(L_{k}\left(\frac{k}{m}\right)\right)\right)<1 / N$ (see figure).

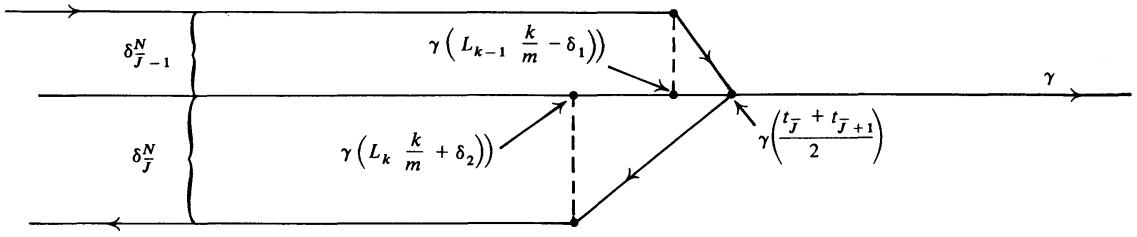

It is easy to see that $\bar{c}_{\bar{\varepsilon}}$ is simple (since $j_{N}=\bar{j}$ ). Also $d\left(\gamma(t), \bar{c}_{\bar{\varepsilon}}(t)\right)<1 / N<\bar{\varepsilon}$ for $t \in[0,1]$. Thus the lemma follows.

Before proving Lemma 2.4 we prove a useful lemma.

Lemma A.2. Let $\gamma_{1}$ and $\gamma_{2}$ be simple curves on $M$. Assume $x, y \in M$ are such that $x \in M^{+}\left(\gamma_{1}\right), y \in M^{-}\left(\gamma_{1}\right)$ and $\gamma_{1}$ is homotopic to $\gamma_{2}$ in $M-\{x, y\}$. Then $x \in M^{+}\left(\gamma_{2}\right)$ and $y \in M^{-}\left(\gamma_{2}\right)$.

Proof. Since the Gauss map is a diffeomorphism, we may assume that $M$ is the unit sphere. For $y \in M$ let $S_{y}$ represent stereographic projection from $y$. Since $y \in M^{-}\left(\gamma_{1}\right)$ and $x \in M^{+}\left(\gamma_{1}\right)$, we see that the winding number of $S_{y}\left(\gamma_{1}\right)$ about $S_{y}(x)$ is +1 . Since $\gamma_{2}$ is homotopic to $\gamma_{1}$ in $M-\{x, y\}, S_{y}\left(\gamma_{2}\right)$ is homotopic to $S_{y}\left(\gamma_{1}\right)$ in $\mathbf{R}^{2}-\left\{S_{y}(x)\right\}$. Hence the winding number of $S_{y}\left(\gamma_{2}\right)$ around $S_{y}(x)$ is +1 . Thus $x \in M^{+}\left(\gamma_{2}\right)$ and $y \in M^{-}\left(\gamma_{2}\right)$.

Proof of Lemma 2.4. Let $\delta_{0}>0$ and $\varepsilon_{0}>0$ be as in the definition of nondegenerate. There is an $\varepsilon>0$ such that $\varepsilon_{0}>\varepsilon$ and such that $\operatorname{Vol}\{x \in M \mid$ $d(x, \gamma) \leqslant \varepsilon\}<\delta_{0} / 2$; we will denote $\{x \in M \mid d(x, \gamma) \leqslant \varepsilon\}$ by $T_{\varepsilon}(\gamma)$. Let $c$ be a 
piecewise smooth simple closed curve such that $d(\gamma(t), c(t))<\varepsilon$ (which exists since $\left.\gamma \in \Omega^{m}(M)\right)$. By the definition of $\delta_{0}$ and the fact that $\varepsilon<\varepsilon_{0}$ and $\operatorname{Vol}\left(T_{\varepsilon}(\gamma)\right)<\delta_{0} / 2$, there are an $x_{0} \in M^{+}(c)-T_{\varepsilon}(\gamma)$ and a $y_{0} \in M^{-}(c)-$ $T_{\varepsilon}(\gamma)$. Now let $x \in M-\gamma$ and let $\bar{\varepsilon}<\min \left\{\varepsilon, \frac{1}{2} d(x, \gamma)\right\}$. Let $\tau_{1}$ and $\tau_{2}$ be two piecewise smooth simple closed curves such that $d\left(\gamma(t), \tau_{i}(t)\right)<\bar{\varepsilon}, i=1,2$. To prove part (a) of the lemma we need only show $x \in M^{+}\left(\tau_{i}\right)$ or $x \in M^{-}\left(\tau_{i}\right)$, $i=1,2$.

We first note that $\tau_{1}$ is homotopic to $\tau_{2}$ inside $T_{\bar{\varepsilon}}(\gamma)$ since they are both homotopic to $\gamma$ inside $T_{\bar{\varepsilon}}(\gamma)$, and further $\tau_{i}(i=1,2)$ is homotopic to $c$ inside $T_{\varepsilon}(\gamma)$. Now Lemma A.2 tells us that $x_{0} \in M^{+}\left(\tau_{i}\right)$ and $y_{0} \in M^{-}\left(\tau_{i}\right)$.

Assume that $x \in M^{+}\left(\tau_{1}\right)$. Since $\tau_{2}$ is homotopic to $\tau_{1}$ in $T_{\bar{\varepsilon}}(\gamma) \subset M-$ $\left\{x, y_{0}\right\}$, we see that $x \in M^{+}\left(\tau_{2}\right)$ (Lemma A.2). Similarly if $x \in M^{-}\left(\tau_{1}\right)$, then $x \in M^{-}\left(\tau_{2}\right)$. Thus we have shown part (a) of the lemma.

For part (b) assume $x, y \in K$ where $K$ is a connected component of $M-\gamma$. Assume further without loss of generality that $x \in M^{+}(\gamma)$. We need to show $y \in M^{+}(\gamma)$. Let $\varepsilon_{0}$ be such that for every simple closed piecewise smooth curve $\sigma$ such that $d(\gamma(t), \sigma(t))<\varepsilon_{0}$ we have $x \in M^{+}(\sigma)$ (this exists by the definition of $M^{+}(\gamma)$ ). Let $\tau \subset M-\gamma$ be a curve from $x$ to $y$, and let $\varepsilon<\min \left\{\varepsilon_{0}, d(\gamma, \tau)\right\}$. We need only show that $y \in M^{+}(\sigma)$ for any piecewise smooth simple closed $\sigma$ such that $d(\gamma(t), \sigma(t))<\varepsilon$. But this is clear, since $\tau \in M-\sigma, x$ and $y$ lie in the same component of $M-\sigma$, and since $\varepsilon<\varepsilon_{0}, x \in M^{+}(\sigma)$ and hence $y \in M^{+}$ $(\sigma)$.

To see part (c) of the lemma it is sufficient to notice that by the previous arguments for sufficiently small $\varepsilon>0, M^{+}(\gamma) \cap\left(M-T_{\varepsilon}(\gamma)\right)=M^{+}\left(c_{\varepsilon}^{\gamma}\right) \cap$ $\left(M-T_{\varepsilon}(\gamma)\right)$ for all $c_{\varepsilon}^{\gamma}$ which approximate $\gamma$ within $\varepsilon$. Now since $\operatorname{Vol}\left(T_{\varepsilon}(\gamma)\right) \rightarrow 0$ as $\varepsilon \rightarrow 0$, and since $M$ is compact and thus $f$ bounded, we have $\int_{M^{+}(\gamma)} f=$ $\lim _{\varepsilon \rightarrow 0} \int_{M^{+} c_{\varepsilon}^{\gamma}} f$.

Part (d) is also similar. Let $F:[0,1] \times[0,1] \rightarrow M$ be the homotopy from $\gamma$ to $\bar{\gamma}$ in $M-\{x, y\}$. Let $\varepsilon<\frac{1}{2} \min \{d(x, F([0,1] \times[0,1])), d(y, F([0,1] \times[0,1]))\}$. Then if $c_{\varepsilon}^{\gamma}$ and $c_{\varepsilon}^{\gamma}$ are $\varepsilon$ approximations to $\gamma$ and $\bar{\gamma}$ respectively, they are homotopic in $M-\{x, y\}$ ( $c_{\varepsilon}^{\gamma}$ is homotopic to $\gamma$ in $M-\{x, y\}$, etc.). Since $x \in M^{+}\left(c_{\varepsilon}^{\gamma}\right)$ and $y \in M^{-}\left(c_{\varepsilon}^{\gamma}\right)$ by Lemma A.2, $x \in M^{+}\left(c_{\varepsilon}^{\gamma}\right)$ and $y \in M^{-}\left(c_{\varepsilon}^{\gamma}\right)$. Since this was true for any choice of $c_{\varepsilon}^{\gamma}$, we have $x \in M^{+}(\bar{\gamma})$ and $y \in M^{-}(\bar{\gamma})$. To see that $\bar{\gamma}$ is nondegenerate, we note that the same argument as for part (b) shows that for all such $c_{\varepsilon}^{\gamma}$ we have $B_{\varepsilon}(x) \subset M^{+}\left(c_{\varepsilon}^{\gamma}\right)$ and $B_{\varepsilon}(y) \subset M^{-}\left(c_{\varepsilon}^{\gamma}\right)$. Thus in the definition of nondegenerate take $\varepsilon$ to be this $\varepsilon$ and $\delta=$ $\min \left\{\operatorname{Vol}\left(B_{\varepsilon}(x)\right), \operatorname{Vol}\left(B_{\varepsilon}(y)\right)\right\}$.

Remarks A.3. (a) Let $\gamma \in \Omega^{m}(M)$ be regular, and let $0(\gamma)>0$ be a number such that there exists $x_{0}, y_{0} \in M-\gamma$ with $x_{0} \in M^{+}(\gamma), y_{0} \in M^{-}(\gamma)$, 
$d\left(y_{0}, \gamma\right)>0(\gamma)$, and $d\left(x_{0}, \gamma\right)>0(\gamma)$. Then the preceding arguments tell us that for $x \in M-\gamma$ and $\delta, 0<\delta<\min \{0(\gamma), d(x, \gamma)\}$, we have $x \in M^{+}(\gamma)$ if and only if $x \in M^{+}\left(c_{\delta}\right)$ for all piecewise smooth simple closed curves $c_{\delta}$ such that $d\left(\gamma(t), c_{\delta}(t)\right)<\delta$. This follows since all such curves are homotopic in $M-\left\{x, y_{0}\right\}$. A similar statement holds for $x \in M^{-}(\gamma)$.

(b) Let $\gamma \in \Omega^{m}(M)$ be regular, $x \in \gamma[0,1]-\gamma\left(\left\{\frac{i}{m} \mid i=1,2, \cdots, m\right\}\right)$, and $V$ a unit vector perpendicular to $\gamma$ at $x$. Let $\varepsilon$ be small for $\gamma$ such that $\varepsilon<$ $d\left(x, \gamma\left(\left\{\frac{i}{m} \mid i=1,2, \cdots, m\right\}\right)\right)$, and let $\delta$ be much smaller than $\varepsilon$ (see the beginning of the appendix). Let $c_{\delta}$ be an approximation to $\gamma$ as in Lemma A.1. Then for each $t \in \gamma^{-1}(x)$ there is a $\delta_{t}>0$ such that $c_{\delta}(t)=\operatorname{Exp}_{x} \delta_{t} V$. Using the fact that $c_{\delta}$ is simple and the definition of $t_{1}>t_{2}$, it is easy to see that $t_{1}>t_{2}$ if and only if $\delta_{t_{1}}>\delta_{t_{2}}$. Thus we can use $\delta_{t_{1}}>\delta_{t_{2}}$ as a definition of $t_{1}>t_{2}$.

It should be noted that $>_{V}$ is only defined when $\gamma$ is regular. In fact this alternate definition has the same restriction as the ordering $\delta_{t_{1}}>\delta_{t_{2}}$ could be reversed for different approximating curves $c_{\delta}$, if $\gamma$ has a $180^{\circ}$ turn.

Lemma A.4. Let $\gamma \in \Omega^{m}(M)$ be regular, and $x \in \gamma[0,1]-\gamma\left\{\frac{i}{m} \mid i=\right.$ $1,2, \cdots, m\}$. Let $t \in \gamma^{-1}(x) \equiv\left\{t_{1}, \cdots, t_{r}\right\}$, and let $V$ be the unit vector at $x$ perpendicular to $\gamma$ such that the orientation given by $\left(\gamma^{\prime}(t), V\right)$ is the orientation of $M$. Let $\tau$ be the unit speed geodesic with initial tangent $V$. Then $t$ is a boundary parameter of some component $K$ of $M^{+}(\gamma)$ (respectively $M^{-}(\gamma)$ ) if and only if $t$ is maximal (resp. minimal) in $\gamma^{-1}(x)$ with respect to $\vec{V}_{V}$. Furthermore if the above holds then $K$ is the component of $M^{+}(\gamma)$ (resp. $\left.M^{-}(\gamma)\right)$ towards which $V$ (resp. $-V$ ) points.

Proof. We will prove the $M^{+}(\gamma)$ case. The $M^{-}(\gamma)$ case is similar.

Let $\bar{K}$ be the component of $M-\gamma$ towards which $V$ points. We need only show, by the definition of boundary parameter, that if $t$ is the boundary parameter of $K \subset M^{+}(\gamma)$ then $K=\bar{K}$, and that if $t$ is maximal with respect to $\underset{V}{>}$ then $\bar{K} \subset M^{+}(\gamma)$.

Assume that $t$ is the boundary parameter of $K \subset M^{+}(\gamma)$, and let $\bar{V}$ be the unit vector perpendicular to $\gamma$ at $x$ pointing towards $K$. We need to show $\bar{V}=V$. Let $\varepsilon$ and $\delta$ satisfy the conditions in Remarks A.3. Let $\bar{\tau}$ be the unit speed geodesic with initial tangent $\bar{V}$. Let $y=\bar{\tau}(\delta)$. Let $c_{\delta}$ be an approximation as in Lemma A.1 and let $\delta_{t}$ be such that $\bar{\tau}\left(\delta_{t}\right)=c_{\delta}(t)$. By the definitions and Remarks A.3 we have $\bar{\tau}(s) \notin \gamma$ and $\bar{\tau}(s) \notin c_{\delta}$ for $\delta_{t}<s \leqslant \delta$, and in particular $y=\bar{\tau}(\delta) \in M^{+}(\gamma) \subset M^{+}\left(c_{\delta}\right)$. Thus the orientation given by $\left(c_{\delta}^{\prime}(t), \bar{\tau}^{\prime}\left(\delta_{t}\right)\right)$ is the same as the orientation of $M$ (by the definition of the + side of a simple curve). On the other hand the orientation given by $\left(\gamma^{\prime}(t), \bar{V}\right)$ is the same as the orientation given by $\left(c_{\delta}^{\prime}(t), \bar{\tau}^{\prime}\left(\delta_{t}\right)\right)$, and hence $\bar{V}=V$. 
Now assume that $t$ is maximal with respect to $>$. Let $\varepsilon, \delta$ and $c_{\delta}$ be as in the previous section. Let $\tau$ be defined by $V$. By the choice of $\varepsilon$ and $\delta, \tau(\delta) \in \bar{K}$. Let $\delta_{t}$ be such that $\tau\left(\delta_{t}\right)=c_{\delta}(t)$. By the maximality of $t$ with respect to $>$ and by Remark A.3(b), for $\delta_{t}<s \leqslant \delta$ we have $\tau(s) \notin c_{\delta}$. Since the orientation given by $\left(\gamma^{\prime}(t), V\right)$ is the orientation of $M$, so is the orientation given by $\left(c_{\delta}^{\prime}(t), \tau^{\prime}\left(\delta_{t}\right)\right)$ and hence $\tau(\delta) \in M^{+}\left(c_{\delta}\right)$. Now by Remark A.3(a) $\tau(\delta) \in M^{+}$ $(\gamma)$, and we see $\bar{K} \subset M^{+}(\gamma)$ since $\tau(\delta) \in \bar{K}$.

Proof of Lemma 2.6. By continuity we need only consider $t$ a boundary parameter for $K \subset M^{+}(\gamma)$, with $\gamma(t) \notin \gamma\left(\left\{\frac{i}{m} \mid i=1, \cdots, m\right\}\right)$. But this follows directly from Lemma A.4. The argument also works for $K \subset M^{-}(\gamma)$.

Lemma A.5. Let $\gamma \in \Omega^{m}(M)$ be regular, and assume that $M^{+}(\gamma)$ (respectively $\left.\mathrm{M}^{-}(\gamma)\right)$ is connected. Then every $t \in[0,1]$ is a boundary parameter for $M^{+}(\gamma)\left(\right.$ resp. $\left.M^{-}(\gamma)\right)$.

Proof. Let $\left\{t_{0}, t_{1}, \cdots, t_{p}\right\}$ be $\gamma^{-1}\left(\gamma\left(\left\{\frac{i}{m} \mid i=1, \cdots, m\right\}\right)\right)$ as at the beginning of the appendix. It is clear, since the set of boundary parameters is closed, that we need only show the result for $t \notin\left\{t_{0}, t_{1}, \cdots, t_{p}\right\}$. It is also clear from the definitions that if for some $t \in\left(t_{i}, t_{i+1}\right), t$ is not a boundary parameter for $M^{+}(\gamma)$, then no element of $\left(t_{i}, t_{i+1}\right)$ is a boundary parameter for $M^{+}(\gamma)$. Let $\bar{t}_{i}=\frac{1}{2}\left(t_{i}+t_{i+1}\right)\left(\bar{t}_{p}=\frac{1}{2}\left(t_{p}+1\right)\right)$ for $i=0,1, \cdots, p$. By the above we need only show that $\bar{t}_{i}$ is a boundary parameter for $M^{+}(\gamma)$ for each $i$.

Assume that some $\bar{t}_{i}$ is not a boundary parameter of $M^{+}(\gamma)$. We will show that $\gamma$ has a $180^{\circ}$ turn contradicting the regularity of $\gamma$. Let $V$ be the unit vector perpendicular to $\gamma$ at $\gamma\left(\bar{t}_{i}\right)$ such that the orientation given by $\left(\gamma^{\prime}\left(\bar{t}_{i}\right), V\right)$ is the orientation of $M$. By Lemma A.4, $\bar{t}_{i}$ is not maximal with respect to $\vec{V}_{V}$. By the definition of the $t_{i}$ and the fact that $\gamma$ is parameterized proportional to arc length on $\left[t_{i}, t_{i+1}\right]$, we see that if $t \in \gamma^{-1}\left(\gamma\left(\bar{t}_{i}\right)\right)$, then $t=\bar{t}_{j}$ for some $j$. Choose $\bar{t}_{j} \in \gamma^{-1}\left(\gamma\left(\bar{t}_{i}\right)\right)$ such that $\bar{t}_{j}>_{V} \bar{t}_{i}$ and such that there is no $t \in \gamma^{-1}\left(\gamma\left(\bar{t}_{i}\right)\right)$ such that $\bar{t}_{j}>_{V} t>_{V} \bar{t}_{i}$. Now considering $c_{\delta}$ for sufficiently small $\delta$ as in Lemma A. 1 and by Remark A.3 we see that the orientation of $\gamma$ at $\bar{t}_{j}$ is opposite to that of $\gamma$ at $\bar{t}_{i}$. In particular since $\bar{t}_{i}>\bar{t}_{j}$ we see that $\bar{t}_{j}$ is also not a boundary parameter of $M^{+}(\gamma)$. Let $S=\left\{t \in\left\{\bar{t}_{0}, \bar{t}_{1}, \cdots, \bar{t}_{p}\right\} \mid t\right.$ is not a boundary parameter of $\left.M^{+}(\gamma)\right\}$. We define a function $f: S \rightarrow S$ by $f\left(\bar{t}_{i}\right)=\bar{t}_{j}$ where $\bar{t}_{j}$ was defined as above. $f$ is a fixed point free involutive function such that $\gamma\left(\bar{t}_{i}\right)=$ $\gamma\left(f\left(\bar{t}_{i}\right)\right)$.

We claim that for all $\bar{t}_{i} \in S$ and for exactly one of the intervals $\left[\bar{t}_{i}, f\left(\bar{t}_{i}\right)\right]$ or [ $\left.f\left(\bar{t}_{i}\right), \bar{t}_{i}\right]$ (here if $f\left(\bar{t}_{i}\right)>\bar{t}_{i}$, then $\left[f\left(\bar{t}_{i}\right), \bar{t}_{i}\right]$ means $\left.\left[f\left(\bar{t}_{i}\right), 1\right] \cup\left[0, \bar{t}_{i}\right)\right)$, call that interval $I\left(\bar{t}_{i}\right)$, we have for any $t_{j} \in\left\{\bar{t}_{0}, \ldots, \bar{t}_{p}\right\}$ if $\bar{t}_{j} \in I\left(\bar{t}_{i}\right)$, then $\bar{t}_{j} \in S$ and $f\left(\bar{t}_{j}\right) \in I\left(\bar{t}_{i}\right)$. 
Before proving the claim we show this proves that $\gamma$ makes a $180^{\circ}$ turn giving the desired contradiction. Choose $\bar{t}_{i} \in S$ so that $I\left(\bar{t}_{i}\right)$ is minimal (i.e., if $\bar{t}_{j} \in S$ and $I\left(\bar{t}_{j}\right) \subset I\left(\bar{t}_{i}\right)$, then $\left.I\left(\bar{t}_{j}\right)=I\left(\bar{t}_{i}\right)\right)$. If $\bar{t}_{j} \in I\left(\bar{t}_{i}\right), \bar{t}_{j} \neq \bar{t}_{i}$ and $\bar{t}_{j} \neq f\left(\bar{t}_{i}\right)$, then $f\left(\bar{t}_{j}\right)$ also satisfies these conditions, hence by the claim $I\left(\bar{t}_{j}\right)$ is strictly contained in $I\left(\bar{t}_{i}\right)$ contradicting the minimality of $I\left(f\left(\bar{t}_{i}\right)\right)$. Hence $f\left(\bar{t}_{i}\right)=\bar{t}_{i+1}$ (or $\bar{t}_{i-1}$ ), and thus $\gamma$ makes a $180^{\circ}$ turn at $t_{i+1}$ (or $t_{i}$ ).

We now prove the claim. Since $\gamma$ is regular, $M^{+}(\gamma)$ is nonempty, and hence there is some boundary parameter interval for $M^{+}(\gamma)$, so both intervals in question cannot have that property. Assume $\bar{t}_{j} \in\left[\bar{t}_{i}, f\left(\bar{t}_{i}\right)\right], \bar{t}_{k} \in\left[f\left(\bar{t}_{i}\right), t_{i}\right]$, and both are boundary parameters for $M^{+}(\gamma)$. Let $V_{j}$ and $V_{k}$ be as in Lemma A.4. Choose $\varepsilon$ and $\delta$ small as in Remarks A.3 and Lemma A.4, and let $y_{j}=\operatorname{Exp} \delta V_{j}$, $y_{k}=\operatorname{Exp} \delta V_{k}$. As in the proof of Lemma A.4, $y_{j}, y_{k} \in M^{+}(\gamma)$ and $y_{j}, y_{k} \in$ $M^{+}\left(c_{\delta}\right)$ for all $\delta$ approximations to $\gamma$. Let $\sigma$ be a curve in $M^{+}(\gamma)$ from $y_{j}$ to $y_{k}$, and let $\bar{\delta}=\frac{1}{2} d(\gamma, \sigma)<\frac{1}{2} \delta$. Let $c_{\bar{\delta}}$ be an approximation as in Lemma A.1. We have $\sigma \subset M^{+}\left(c_{\bar{\delta}}\right)$. Let $\tau$ be the minimizing geodesic segment from $c_{\delta}\left(\bar{t}_{i}\right)$ to $c_{\delta}\left(f\left(\bar{t}_{i}\right)\right)$, and let $\tau_{1}$ be the simple closed curve given by $\tau \cup c_{\delta}\left[\bar{t}_{i}, f\left(\bar{t}_{i}\right)\right]$ and $\tau_{2}$ the simple closed curve given by $\tau \cup c_{\delta}\left[f\left(t_{i}\right), t_{i}\right]$. The orientation on $\tau_{i}$ is chosen to agree with that of $c_{\bar{\delta}}$. It is easy to see that $M^{+}\left(\tau_{1}\right) \cup M^{+}\left(\tau_{2}\right)=M^{+}$ $\left(c_{\delta}\right)-\tau, M^{+}\left(\tau_{1}\right) \cap M^{+}\left(\tau_{2}\right)=\varnothing$ and $y_{j} \in M^{+}\left(\tau_{1}\right), y_{k} \in M^{+}\left(\tau_{2}\right)$. Since the curve $\sigma \subset M^{+}\left(c_{\delta}\right)$ goes from $y_{j}$ to $y_{k}$, we see that $\sigma$ intersects $\tau$ but that implies that $\sigma$ comes within $\bar{\delta}$ of $\gamma\left(\bar{t}_{i}\right)$ contradicting the definition of $\bar{\delta}$. Thus we have shown that one of these intervals, called $I\left(\bar{t}_{i}\right)$, has the property that if $\bar{t}_{j} \in I\left(\bar{t}_{i}\right)$ then $\bar{t}_{j} \in S$.

We now need to show that $f\left(\bar{t}_{j}\right) \in I\left(\bar{t}_{i}\right)$. Choose $\varepsilon$ and $\delta$ small as in Remarks A.3 and Lemma A.4, let $c_{\delta}$ be as in Lemma A.1, and let $\tau_{1}$ and $\tau_{2}$ be as in the previous paragraph (we assume $\tau_{1}$ corresponds to $I\left(\bar{t}_{i}\right)$ ). Let $V$ be the unit vector at $\gamma\left(\bar{t}_{j}\right)$, perpendicular to $\gamma$ and such that the orientation given by $\left(\gamma^{\prime}\left(t_{j}\right), V\right)$ is that of $M$. Let $\bar{\tau}$ be the unit speed geodesic determined by $V$. Let $\delta_{1}$ and $\delta_{2}$ be such that $\bar{\tau}\left(\delta_{1}\right)=c_{\delta}\left(\bar{t}_{j}\right)$ and $\bar{\tau}\left(\delta_{2}\right)=c_{\delta}\left(f\left(\bar{t}_{j}\right)\right)$. By the definition of $f$ and Remarks A.3 we have $\bar{\tau}(s) \in M-c_{\delta}$ for $s \in\left(\delta_{1}, \delta_{2}\right)$. By the choice of $V$ and $\tau_{1}$ we see that $\bar{\tau}(s) \in M^{+}\left(\tau_{1}\right)$ for $s \in\left(\delta_{1}, \delta_{2}\right)$. This says that $\bar{\tau}\left(\delta_{2}\right) \in \partial M^{+}$ $\left(\tau_{1}\right)$ so that $c_{\delta}\left(f\left(\bar{t}_{j}\right)\right)=\bar{\tau}\left(\delta_{2}\right) \in c_{\delta}\left(I\left(\bar{t}_{i}\right)\right)$ and hence $f\left(\bar{t}_{j}\right) \in I\left(\bar{t}_{i}\right)$. Thus the claim and hence the lemma are proved.

Let $\gamma \in \Omega^{m}(M)$ be regular, and let $K \subset M^{+}(\gamma)$ (resp. $M^{-}(\gamma)$ ) be a component which is not a cul-de-sac, and let $[a, b]$ and $[c, d]$ be two disjoint maximal intervals of parameter values for $K$ (such intervals exist since $K$ is not a cul-de-sac). Choose $t_{0} \in(a, b)$ and $t_{1} \in(c, d)$ such that $t_{0}, t_{1} \in\left\{\bar{t}_{0}, \bar{t}_{1}, \cdots, \bar{t}_{p}\right\}$ where the $\bar{t}_{i}$ we defined at the beginning of the proof of Lemma A.5. Then there exists a simple piecewise geodesic $\tau:[0,1] \rightarrow M$, which will be called a 
separating curve for $K$, such that

(a) $\tau(0,1) \subset K \subset M-\gamma$,

(b) $\tau(0)=\gamma\left(t_{0}\right)$ and $\tau(1)=\gamma\left(t_{1}\right)$,

(c) after reparameterization the closed piecewise geodesics $\tau_{1}=\tau \cup \gamma\left[t_{0}, t_{1}\right]$ and $\tau_{2}=\tau \cup \gamma\left[t_{1}, t_{0}\right]$ (the orientation chosen to agree with that of $\gamma$ ) are regular elements of $\Omega^{m_{1}}(M)$ and $\Omega^{m_{2}}(M)$ respectively.

(d) if $x \in M^{-}(\gamma)$ then $x \in M^{-}\left(\tau_{i}\right), i=1,2$, and if $x \in M^{+}(\gamma)-\tau$, then $x \in M^{+}\left(\tau_{i}\right)$ and $x \in M^{-}\left(\tau_{j}\right)$ for $i \neq j$ and $i=1$ or 2 .

Define $\tau$ to start at $\gamma\left(t_{0}\right)$ orthogonal to $\gamma$, being a geodesic to length $\delta_{0}$, and end at $\gamma\left(t_{1}\right)$ orthogonal to $\gamma$, the last geodesic segment having length $\delta_{1}$, such that between it lies inside $K$ and is defined to have breaks in such a way that each geodesic segment has length $\leqslant c$.

Properties (a) and (b) are immediate from the definition of $\tau$. To see properties (c) and (d) we need to approximate the curves $\tau_{i}$ by simple curves. Let $\varepsilon$ and $\delta$ be as in Remarks A.3 and Lemma A.4. Now for every $\bar{\delta}$ such that $\bar{\delta}<\min \left\{\delta, \delta_{0}, \delta_{1}\right\}$ we define approximating curves $c_{\delta}^{i}$ to $\tau_{i}$ as follows: Let $c_{\delta}$ be an approximation to $\gamma$ as in Lemma A.1, and construct $c_{\delta}^{i}$ by joining $c_{\delta}\left(t_{0}\right)$ to $c_{\delta}\left(t_{1}\right)$ via $\tau$ (the joining curve may be a slight extension of $\tau$ or not all of $\tau$; see Fig. A.1). It is easily seen that by choosing an appropriate parameter for $c_{\delta}^{i}$ it is a $\bar{\delta}$ approximation to $\tau_{i}$.
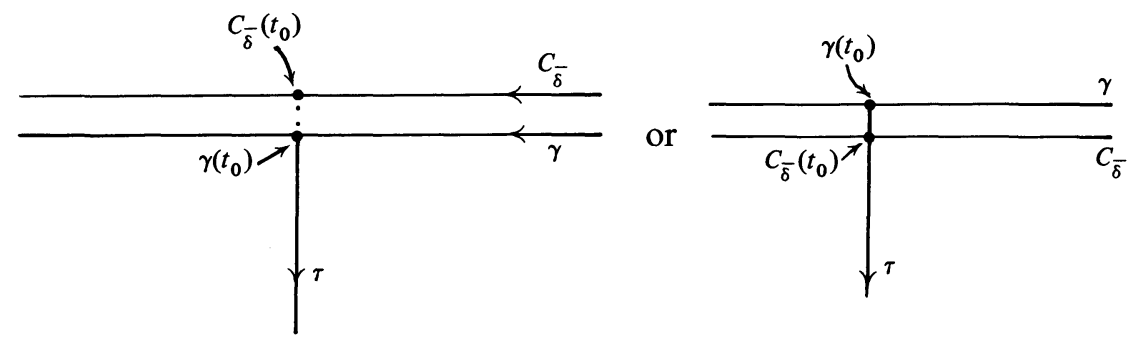

\section{FIG A.1}

Since we can construct $c_{\delta}^{i}$ for all sufficiently small $\bar{\delta}$, we have that $\tau_{i} \in$ $\Omega^{m_{i}}(M)$ for some $m_{i}$. For all such $\bar{\delta}$ it is easy to see that $M^{+}\left(c_{\delta}^{-}\right)-\tau$ (or the slight extension of $\tau)=M^{+}\left(c_{\delta}^{1}\right) \cup M^{+}\left(c_{\delta}^{2}\right)$ and $M^{+}\left(c_{\delta}^{1}\right) \cap M^{+}\left(c_{\delta}^{2}\right)=\varnothing$. Also since $\tau$ splits $K$ into two pieces, let $x_{1}$ and $x_{2}$ be in different pieces of $K-\tau$, and choose $x \in M^{-}(\gamma)$. Since above is true for all small $\bar{\delta}$ it is easy to see that $M^{+}(\gamma)-\tau=M^{+}\left(\tau_{1}\right) \cup M^{+}\left(\tau_{2}\right), M^{+}\left(\tau_{1}\right) \cap M^{+}\left(\tau_{2}\right)=\varnothing, M^{-}(\gamma)$ $=M^{-}\left(\tau_{1}\right) \cap M^{-}\left(\tau_{2}\right)$, and $M^{+}\left(\tau_{1}\right) \neq \varnothing, M^{+}\left(\tau_{2}\right) \neq \varnothing$ since $x_{1} \in M^{+}\left(\tau_{1}\right)$ and $x_{2} \in M^{+}\left(\tau_{2}\right)$. Thus property (d) follows. 
To see that $\tau_{i}$ is regular we note that by the definition of $\tau$ we have no $180^{\circ}$ turns in $\tau_{i}$ ( $\tau$ is simple and orthogonal to $\gamma$ ), and also all segments of $\tau$ have nonzero length. To see that $\tau_{i}$ is nondegenerate we simply note that $x_{i} \in M^{+}\left(\tau_{i}\right)$ and $y \in M^{+}\left(\tau_{i}\right)$ and see that the proof of Lemma 2.4 shows that $\tau_{i}$ is nondegenerate.

Lemma A.6. Let $\gamma \in \Omega^{m}(M)$ be regular, and $K \subset M^{+}(\gamma)\left(\right.$ resp. $\left.M^{-}(\gamma)\right)$ be a component which is not a cul-de-sac. Let $\tau$ be a separating curve of $K$. Let $\left\{K, K_{1}, \cdots, K_{r}\right\}$ be the components of $M^{+}(\gamma)$ (resp. $\left.M^{-}(\gamma)\right)$. Then for $i=1,2$, $M^{+}\left(\tau_{i}\right)$ is not connected, $M^{+}\left(\tau_{1}\right) \cap M^{+}\left(\tau_{2}\right)=\varnothing, M^{+}\left(\tau_{1}\right) \cup M^{+}\left(\tau_{2}\right)=M^{+}$ $(\gamma)-\tau$. Further if $\bar{K}$ is a component of $\tau_{i}$, then $\bar{K} \subset K$ or $\bar{K} \in\left\{K_{1}, \cdots, K_{r}\right\}$, and if $\bar{K} \pitchfork K$ is a cul-de-sac of $\tau_{i}$, then $\bar{K}$ is a cul-de-sac of $\gamma$.

Proof. It follows directly from Property (d) of separating curves that $M^{+}\left(\tau_{1}\right) \cap M^{+}\left(\tau_{2}\right)=\varnothing$ and $M^{+}\left(\tau_{1}\right) \cup M^{+}\left(\tau_{2}\right)=M^{+}(\gamma)-\tau$. It is also clear that if $\bar{K}$ is a connected component of $M^{+}\left(\tau_{i}\right)$, then $\bar{K} \subset K$ or $\bar{K} \in$ $\left\{K_{1}, \cdots, K_{r}\right\}$.

Using the arguments similar to those in Lemma A.4, and considering the approximating curves $c_{\delta}^{i}$ it is not hard to see that if $\bar{K}$ is a component of $M^{+}\left(\tau_{1}\right)$, then $t$ is a boundary parameter for $\bar{K}$ (if $\bar{K} \subset K$ we mean $K$ here) as a component of $M^{+}(\gamma)$ if and only if $t \in\left[t_{0}, t_{1}\right]$ and the corresponding parameter of $\tau_{1}$ is a boundary parameter of $\bar{K}$ as a component of $M^{+}\left(\tau_{1}\right)$. Similarly for $\tau_{2}$.

The above shows that if $\bar{K} \subset M^{+}\left(\tau_{i}\right)$ is a component such that $\bar{K} \nsubseteq K$ and $\bar{K}$ is a cul-de-sac of $M^{+}\left(\tau_{i}\right)$, then $\bar{K}$ is a cul-de-sac of $M^{+}(\gamma)$.

The only thing left to show is that $M^{+}\left(\tau_{i}\right)$ is not connected. Assume $M^{+}\left(\tau_{1}\right)$ is connected. Then $M^{+}\left(\tau_{1}\right) \subset K$. By Lemma A.5 and the preceding statements we see that every $t \in\left[t_{0}, t_{1}\right]$ is a boundary parameter for $K$ as a component of $M^{+}(\gamma)$. But $t_{0}$ and $t_{1}$ were chosen in disjoint maximal boundary parameter intervals of $K$, giving the desired contradiction.

Proof of Lemma 2.7. We prove this by induction on the number of components of $M^{+}(\gamma)$.

Assume $M^{+}(\gamma)$ has two components $K_{1}$ and $K_{2}$. If both $K_{1}$ and $K_{2}$ are cul-de-sacs, there is nothing to show. Assume $K_{1}$ is not a cul-de-sac, and let $\tau$ be a separating curve for $K_{1}$. Let $\tau$ separate $K_{1}$ into two components $K_{1}^{1}$ and $K_{1}^{2}$. By Lemma A.6 each $M^{+}\left(\tau_{i}\right), i=1,2$, has at least two components in $\left\{K_{1}^{1}, K_{1}^{2}, K_{2}\right\}$. But since this set has only three elements and $M^{+}\left(\tau_{1}\right) \cap M^{+}$ $\left(\tau_{2}\right)=\varnothing$, we get a contradiction.

Assume the lemma is true for all $m$ and all regular $\gamma \in \Omega^{m}(M)$ having fewer than $n>2$ components in $M^{+}(\gamma)$. Let $\gamma \in \Omega^{m}(M)$ be regular such that $M^{+}(\gamma)$ has $n$ components. Since $n>2$, we may assume that there is a 
component $K \subset M^{+}(\gamma)$ which is not a cul-de-sac. Let $\tau$ be a separating curve for $K$. By the definition of $\tau$ and Lemma A.6, $\tau_{i} \in \Omega^{m_{i}}(M)$ is regular, and $M^{+}\left(\tau_{i}\right)$ has fewer than $n$ and at least 2 components. Thus by the induction assumption for $i=1,2$ there are at least two cul-de-sac's in $M^{+}\left(\tau_{i}\right)$, and by Lemma A.6 for each $i=1,2$ at least one of these cul-de-sacs is a cul-de-sac in $M^{+}(\gamma)$. Since $M^{+}\left(\tau_{1}\right) \cap M^{+}\left(\tau_{2}\right)=\varnothing$, there are at least two distinct cul-desacs in $M^{+}(\gamma)$. The result now follows.

Lemma A.7. Let $\gamma \in \Omega^{m}(M)$ be regular, and $K$ a connected component of $M^{+}(\gamma)$. Let $\left\{t_{0}, t_{1}, \cdots, t_{p}\right\}$ be as in the beginning of the appendix (i.e., $\left.\left\{t_{0}, t_{1}, \cdots, t_{p}\right\}=\gamma^{-1}\left(\gamma\left(\left\{\frac{i}{m} \mid i=0,1, \cdots,\right\}\right)\right)\right)$. Assume that for some $i,\left[t_{i-1}, t_{i+1}\right]$ is a boundary parameter interval for $K$, and that $j \neq i$ is such that $\gamma\left(t_{j}\right)=\gamma\left(t_{i}\right)$. Let $\gamma^{k}=\left.\gamma\right|_{\left[t_{k-1}, t_{k}\right]}$ for $k \in\{0,1, \cdots, p\}$. Then $\left(\gamma^{j}\right)^{\prime}\left(t_{j}\right)$ does not lie between $\left(\gamma^{i+1}\right)^{\prime}\left(t_{i}\right)$ and $-\left(\gamma^{i}\right)^{\prime}\left(t_{i}\right)$, where between means a counter clockwise sense as defined by the orientation of M. A similar statement holds for $K \subset M^{-}(\gamma)$.

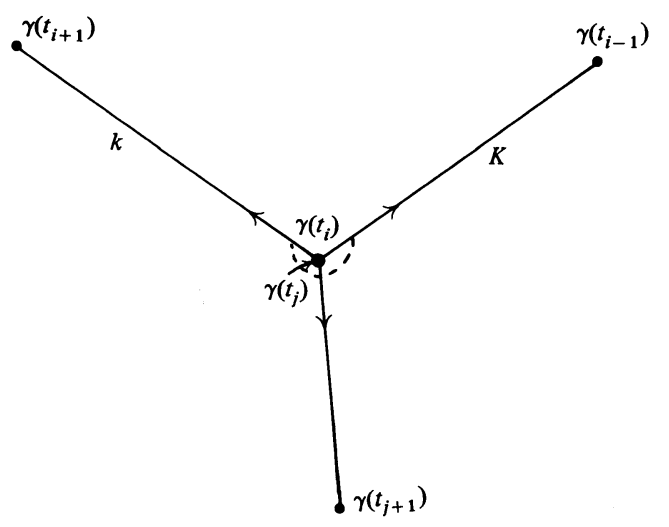

FIG. A.2

(This does not happen)

Proof. Assume $\left(\gamma^{j}\right)^{\prime}\left(t_{j}\right)$ is between $\left(\gamma^{i+1}\right)^{\prime}\left(t_{i}\right)$ and $-\left(\gamma^{i}\right)^{\prime}\left(t_{i}\right)$. Let $\bar{t}_{i-1}$ $=\frac{1}{2}\left(t_{i-1}+t_{i}\right)$ and $\bar{t}_{i}=\frac{1}{2}\left(t_{i}+t_{i+1}\right)$, and $V_{i-1}$ and $V_{i}$ be the unit vectors at $\gamma\left(\bar{t}_{i-1}\right)$ and $\gamma\left(\bar{t}_{i}\right)$ perpendicular to $\gamma$ and such that the orientation given by $\left(\gamma^{\prime}\left(\bar{t}_{i-1}\right), V_{i-1}\right)$ and $\left(\gamma^{\prime}\left(\bar{t}_{i}\right), V_{i}\right)$ is that of $M$.

Choose $\delta_{0}$ so small that

(a) $\operatorname{Exp}\left(s V_{i-1}\right) \in K$ and $\operatorname{Exp}\left(s V_{i}\right) \in K$ for all $0<s \leqslant \delta_{0}$,

(b) the minimizing geodesics from $\gamma\left(t_{i}\right)$ to $\operatorname{Exp}\left(s V_{i-1}\right)$ and $\operatorname{Exp}\left(s V_{i}\right)$ lie in $K$ (except for the point $\left.\gamma\left(t_{i}\right)\right)$ for $0<s<\delta_{0}$.

Such a $\delta_{0}$ exists, since $\left[t_{i-1}, t_{i+1}\right]$ is a boundary parameter of $K$ (see Lemma A.4), and $\gamma$ has only a finite number of geodesic segments, and since all geodesic segments have length less than $c$. 
Let $x=\operatorname{Exp}\left(\delta_{0} \cdot V_{i-1}\right), y=\operatorname{Exp}\left(\delta_{0} \cdot V_{i}\right)$, and let $\tau_{1}$ be the minimizing geodesic from $x$ to $\gamma\left(t_{i}\right)$, and $\tau_{2}$ the minimizing geodesic from $\gamma\left(t_{i}\right)$ to $y$. Since $K$ is connected, so is $K-\left(\tau_{1} \cup \tau_{2}\right)$. Thus let $\tau_{3}$ be a simple curve in $K-\left(\tau_{1} \cup \tau_{2}\right)$ from $x$ to $y$, and $\tau$ the simple closed curve $\tau_{1} \cup \tau_{3} \cup \tau_{2} . \tau$ intersects $\gamma$ only at $\gamma\left(t_{i}\right)$. By construction $\gamma\left[t_{i-1}, t_{i}\right)$ and $\gamma\left(t_{i}, t_{i+1}\right]$ lie in $M^{+}(\tau)$, while $\gamma\left(t_{j}, t_{j+1}\right]$ lies in $M^{-}(\tau)$. We assume without loss of generality that $t_{j}>t_{i}$.

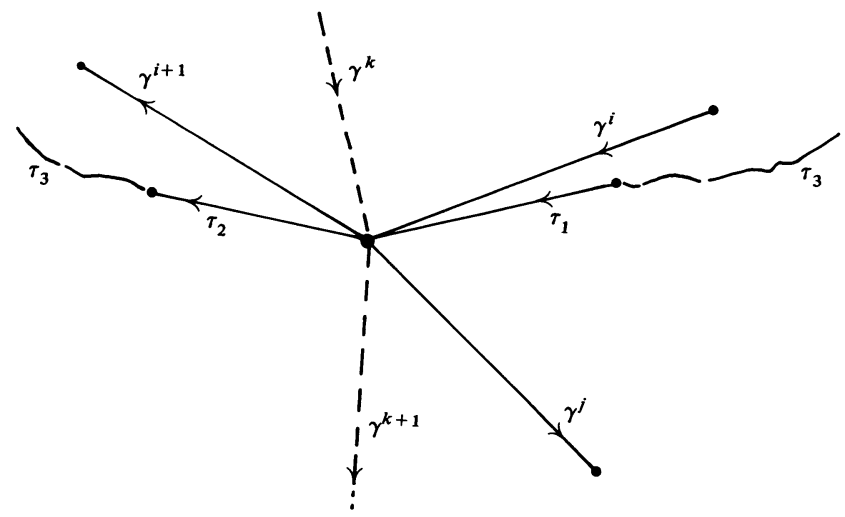

FIG. A.3

Let $\bar{t}=\sup \left\{t \mid \gamma(s) \in\left(M^{+}(\tau)\right)\right.$ for all $\left.s \in\left[t_{i}, t\right]\right\}$, where $\left(M^{+}(\tau)\right)$ is the closure. Clearly $\gamma(\bar{t})=\gamma\left(t_{i}\right)$, hence $\bar{t}=t_{k}$ for some $k \in\{0,1, \cdots, p\}$. By the definition of $t$ and $\tau$ we have $\gamma\left(\left[t_{k-1}, t_{k}\right)\right) \subset M^{+}(\tau)$ and $\gamma\left(\left(t_{k}, t_{k+1}\right]\right) \subset M^{-}$ $(\tau)$. If $\gamma\left[t_{k-1}, t_{k}\right]$ coincides with $\gamma\left(\left[t_{i-1}, t_{i}\right]\right.$ or $\gamma\left[t_{i}, t_{i+1}\right]$, then the fact that $\gamma\left(\left(t_{k}, t_{k+1}\right]\right) \subset M^{-}(\gamma)$ contradicts the fact that $\bar{t}_{i}$ and $\bar{t}_{i-1}$ are maximal with respect to $\vec{V}_{i}$ and $\underset{V_{i-1}}{>}$ respectively. On the other hand by the construction of $\tau$ we see $-\left(\gamma^{k}\right)^{\prime}\left(t_{k}\right)$ lies between $-\left(\gamma^{i}\right)^{\prime}\left(t_{i}\right)$ and $\left(\gamma^{i+1}\right)^{\prime}\left(t_{i}\right)$, while $\left(\gamma^{k+1}\right)^{\prime}\left(t_{k}\right)$ lies between $\left(\gamma^{i+1}\right)^{\prime}\left(t_{i}\right)$ and $\left(\gamma^{i}\right)^{\prime}\left(t_{i}\right)$ and hence $\gamma$ cannot be the limit of simple curves. This contradiction gives the lemma.

Proof of Lemma 2.8. We first show part (a). Since $M^{+}(\gamma)$ is connected, Lemma A.5 says that all $t \in[0,1]$ are boundary parameter values for $M^{+}(\gamma)$, and hence all + vertices are free. To show the second part we need only note that since $\gamma$ is connected, each component of $M-\gamma$ is simply connected, and then apply Gauss-Bonnet to $M^{+}(\gamma)$. There are many ways to see that one can apply Gauss-Bonnet even though $\gamma$ may not be simple. One way is to consider the closed simple piecewise geodesic curves $\gamma_{\varepsilon}$, for sufficiently small $\varepsilon$, defined by $\gamma_{\varepsilon}(i / m)=\tau_{i}(\varepsilon)$ where $\tau_{i}$ is the unit speed geodesic with initial tangent vector half way between (in the counter clockwise sense) $\left(\gamma^{i+1}\right)^{\prime}\left(\frac{i}{m}\right)$ and $-\left(\gamma^{i}\right)^{\prime}\left(\frac{i}{m}\right)$. Now for $\varepsilon>0$ sufficiently small using Lemma A.7, the fact that $l_{i} \leqslant c$, and 
standard convexity arguments, one can obtain that (a) $\gamma_{\varepsilon}$ is simple, (b) $M^{+}\left(\gamma_{\varepsilon}\right) \subset M^{+}(\gamma)$, (c) $\lim _{\varepsilon \rightarrow 0} \gamma_{\varepsilon}=\gamma$, and (d) $\lim _{\varepsilon \rightarrow 0} M^{+}\left(\gamma_{\varepsilon}\right)=M^{+}(\gamma)$. Thus one can apply Gauss-Bonnet to $\gamma_{\varepsilon}$ and letting $\varepsilon \rightarrow 0$ to $\gamma$.

We now show part (b). We first need to show that $\gamma(a)=\gamma(b)$. Let $\left\{t_{0}, \cdots, t_{p}\right\}=\gamma^{-1} \gamma\left(\left\{\frac{i}{m} \mid i=0, \cdots, m-1\right\}\right)$ and $\gamma^{k}=\left.\gamma\right|_{\left[t_{k-1}, t_{k}\right]}$ as usual. We have $a=t_{i}$ and $b=t_{j}$ for some $i$ and $j$ in $\{0, \cdots, p\}$; otherwise the boundary parameters of $K$ would form a larger interval. Let $\left.S=\left\{k \mid \gamma_{i} t_{k}\right)=\gamma\left(t_{i}\right)\right\}$, and for each $k \in S$ let $V_{k}^{+}$and $V_{k}^{-}$be the unit vectors at $\gamma\left(t_{k}\right)$ defined by

$$
V_{k}^{+}=\frac{\left(\gamma^{k+1}\right)^{\prime}\left(t_{k}\right)}{\left|\left(\gamma^{k+1}\right)^{\prime}\left(t_{k}\right)\right|}, \quad V_{k}^{-}=\frac{-\left(\gamma^{k}\right)^{\prime}\left(t_{k}\right)}{\left|\left(\gamma^{k}\right)^{\prime}\left(t_{k}\right)\right|} \text {. }
$$

Let $\bar{V} \in\left\{V_{k}^{+}\right.$or $\left.V_{k}^{-} \mid k \in S\right\}$ be the vector making the smallest nonzero angle (in the counterclockwise sense) with $V_{i}^{+}$. Since $\left.\gamma\right|_{\left[t_{i}, t_{i+1}\right]} \in \partial K$, if $\bar{V}=V_{k}^{-}$ (or $V_{k}^{+}$), then $\left.\gamma\right|_{\left[t_{k-1}, t_{k}\right]}\left(\right.$ or $\left.\gamma\right|_{\left[t_{k}, t_{k+1}\right]}$ ) is contained in the boundary of $K$. Thus we can assume (by choosing an appropriate representative for $\bar{V}$ ) that $\bar{V}=V_{k}^{-}$ where $\left[t_{k-1}, t_{k}\right]$ is a boundary parameter interval for $K$ (by orientation consideration it must be $V_{k}^{-}$and not $V_{k}^{+}$). We also note here that $V_{k}^{-} \neq V_{i}^{+}$ (representing an angle $2 \pi \neq 0$ ), for this would imply $V_{i}^{-}=V_{i}^{+}$, and hence $\gamma$ would make a $180^{\circ}$ turn at $t_{i}$. Since $\left[t_{k-1}, t_{k}\right]$ is a boundary parameter for $K$, $\left[t_{k-1}, t_{k}\right] \subset[a, b]$.

We claim that $b=t_{k}$ (i.e., $j=k$ ). Assume not. Then $\left[t_{k-1}, t_{k+1}\right] \subset[a, b]$, and hence $\left[t_{k-1}, t_{k+1}\right]$ is a boundary parameter for $K$. By the definition of $\bar{V}\left(=V_{k}^{-}\right)$we have $V_{k}^{+}$is not between $V_{i}^{+}$and $V_{k}^{-}$. Further $V_{k}^{+} \neq V_{k}^{-}$, since $\gamma$ makes no $180^{\circ}$ turns, and $V_{k}^{+} \neq V_{i}^{+}$, since then both $\left[t_{k}, t_{k+1}\right]$ and $\left[t_{i}, t_{i+1}\right]$ could not be boundary parameters for $K$ (see Lemma A.4). Thus we arrive at a contradiction to Lemma A.7. Hence $b=t_{k}$.

Since $b=t_{k}$, we have $\gamma(b)=\gamma(a)$. Further since $V_{i}^{+} \neq V_{j}^{-}$, the exterior angle $A$ satisfies $-\pi<A<\pi$. Also since no $V_{k}^{+}$or $V_{k}^{-}$lies between $V_{i}^{+}$and $V_{j}^{-}$, we can define approximations $\bar{\gamma}_{\varepsilon}$, as in part (a), to the closed piecewise geodesic $\left.\gamma\right|_{[a, b]}$. Thus $\gamma$ is the limit of simple curves. It is easy to see that $M^{+}(\gamma)=K$ since each $t \in[a, b]$ is a boundary parameter for $K$, and that $\left.\gamma\right|_{[a, b]}$ is regular. The Gauss-Bonnet formula follows now from part (a).

To see that one of $a$ and $b$ is in fact $i / m$ for some $i=0,1, \cdots, m-1$ one need only note that since $V_{i}^{+} \neq V_{j}^{-}$we cannot have both $V_{i}^{-}=-V_{i}^{+}$and $V_{j}^{+}=-V_{j}^{-}$, for then $\gamma$ would intersect itself transversely at $\gamma(a)=\gamma(b)$ and hence not be the limit of simple curves. Thus the exterior angle at either $a$ or $b$ must be nonzero, and hence one of $a$ and $b$ is a vertex of $\gamma$. Assume $a=i / m$ and let $j$ be as in the lemma. If $i<k<j$, then we have $\left[\frac{k-1}{m}, \frac{k+1}{m}\right]$ is a boundary parameter interval for $K \subset M^{+}(\gamma)$. Hence if $k$ is a + vertex, $k$ is a 
free + vertex. If $j / m \neq b$, then $\left[\frac{j-1}{m}, b\right]$ is a boundary parameter interval for $K$. Since $b>j / m$, if $j$ is a + vertex, then it is a half free + vertex. Thus the lemma is shown.

Proof of Lemma 2.9. Assume $\left[\frac{i-1}{m}, \frac{i}{m}+\varepsilon\right]$ is the boundary parameter for a component $K \subset M^{+}(\gamma)$. The other case is similar.

We claim that for $\varepsilon$ sufficiently small, the minimizing geodesic segment $\tau_{\varepsilon}$ : $\left[\frac{i-1}{m}, \frac{i}{m}\right] \rightarrow M$ from $\gamma\left(\frac{i-1}{m}\right)$ to $\gamma\left(\frac{i}{m}+\varepsilon\right)$ lies inside $K$ (except for $\tau\left(\frac{i-1}{m}\right)$ and $\tau\left(\frac{i}{m}\right)$ ). To see this we first note that since $i$ is a + vertex (and $l_{i}<c$ ) for small $\varepsilon$, the angle $\theta_{\varepsilon}$ from $\gamma^{\prime}\left(\frac{i-1}{m}\right)$ to $\tau^{\prime}\left(\frac{i-1}{m}\right)$ (measured counterclockwise) is small and positive. Thus if $\varepsilon$ is small enough, we can assume that $\theta_{\varepsilon}$ is smaller than any positive angle from $\gamma^{\prime}\left(\frac{i-1}{m}\right)$ to $\gamma^{\prime}(t)$ for any $t$ such that $\gamma(t)=\gamma\left(\frac{i-1}{m}\right)$. Hence if $\tau_{\varepsilon}\left(\frac{i-1}{m}, \frac{i}{m}\right)$ intersects $\gamma$ for arbitrarily small $\varepsilon>0$, we see, using the fact that $\gamma$ has only a finite number of geodesic segments, that there is a segment $\gamma[c, d]$ such that $\gamma(c) \in \gamma\left(\frac{i-1}{m}, \frac{i}{m}\right]$ and $\gamma^{\prime}(c)$ lies between $\gamma^{\prime}(t)$ and $-\gamma^{\prime}(t)$ (in the counterclockwise sense) where $t \in\left(\frac{i-1}{m}, \frac{i}{m}\right]$ is such that $\gamma(t)=$ $\gamma(c)$ (if $t=\frac{i}{m}$ then $\gamma^{\prime}(t)$ is to be interpreted as $\left(\gamma^{i+1}\right)^{\prime}(t)$ and $-\gamma^{\prime}(t)$ as $\left.-\left(\gamma^{i}\right)^{\prime}(t)\right)$. But this contradicts Lemma A.7.

Thus for $\varepsilon$ sufficiently small $\tau_{\varepsilon}\left(\frac{i-1}{m}, \frac{i}{m}\right) \subset K$. It is also clear, from the fact that $l_{i}<c$ and $\operatorname{Ext}(i)>A$, that for $\varepsilon$ small enough $\bar{\gamma}_{\varepsilon}$ satisfies $l_{i}\left(\bar{\gamma}_{\varepsilon}\right)<c$ and $\operatorname{Ext}(\bar{i})>A$.

Choose $\varepsilon_{0}$ so small that for $\varepsilon \leqslant \varepsilon_{0}$ all of the above holds and further $\theta_{\varepsilon}<\pi / 2$.

Every part of the lemma is now clear for $\bar{\gamma}_{\varepsilon}\left(\varepsilon \leqslant \varepsilon_{0}\right)$ except that $\bar{\gamma}_{\varepsilon}$ is the limit of simple curves.

Fix $\varepsilon<\varepsilon_{0}$ and $\delta>0$. We need to find a simple piecewise smooth closed curve $\bar{c}_{\delta}$ such that for all $t \in[0,1]$ we have $d\left(\bar{c}_{\delta}(t), \bar{\gamma}_{\varepsilon}(t)\right)<\delta$. To do this choose the following numbers (see Fig. A.4):

$$
\begin{aligned}
& t^{0}<t^{1}<t^{2}<t^{3}<t^{4}<t^{5} \\
& s^{0}<s^{1}<s^{2}<s^{3}<s^{4}<s^{5}
\end{aligned}
$$

where $s^{0}=t^{0}=\frac{i-1}{m}, s^{2}=t^{3}=\frac{i}{m}, s^{5}=t^{5}=\frac{i+1}{m}, \gamma\left(s^{3}\right)=\bar{\gamma}_{\varepsilon}\left(t^{3}\right)=\bar{\gamma}_{\varepsilon}\left(\frac{i}{m}\right)$, and $\gamma\left(s^{4}\right)=\bar{\gamma}_{\varepsilon}\left(t^{4}\right)$. We further assume they were chosen to have the following properties:

(a) $\gamma\left(s^{1}\right), \bar{\gamma}_{\varepsilon}\left(t^{1}\right)$, and $\gamma\left(s^{0}\right)=\bar{\gamma}_{\varepsilon}\left(t^{0}\right)=\gamma\left(\frac{i}{m}\right)$ have pairwise distances less than $\delta / 4$, and the minimal geodesic $\tau$ from $\gamma\left(s^{1}\right)$ to $\bar{\gamma}_{\varepsilon}\left(t^{1}\right)$ is perpendicular to $\gamma$ at $\gamma\left(s^{1}\right)$. (We can do this since $\theta_{\varepsilon}<\pi / 2$.)

(b) $\gamma\left(s^{3}\right)=\bar{\gamma}_{\varepsilon}\left(t^{3}\right)=\bar{\gamma}_{\varepsilon}\left(\frac{i}{m}\right), \bar{\gamma}_{\varepsilon}\left(t^{2}\right)$, and $\gamma\left(s^{4}\right)=\bar{\gamma}_{\varepsilon}\left(t^{4}\right)$ have pairwise distances less than $\delta / 4$. 


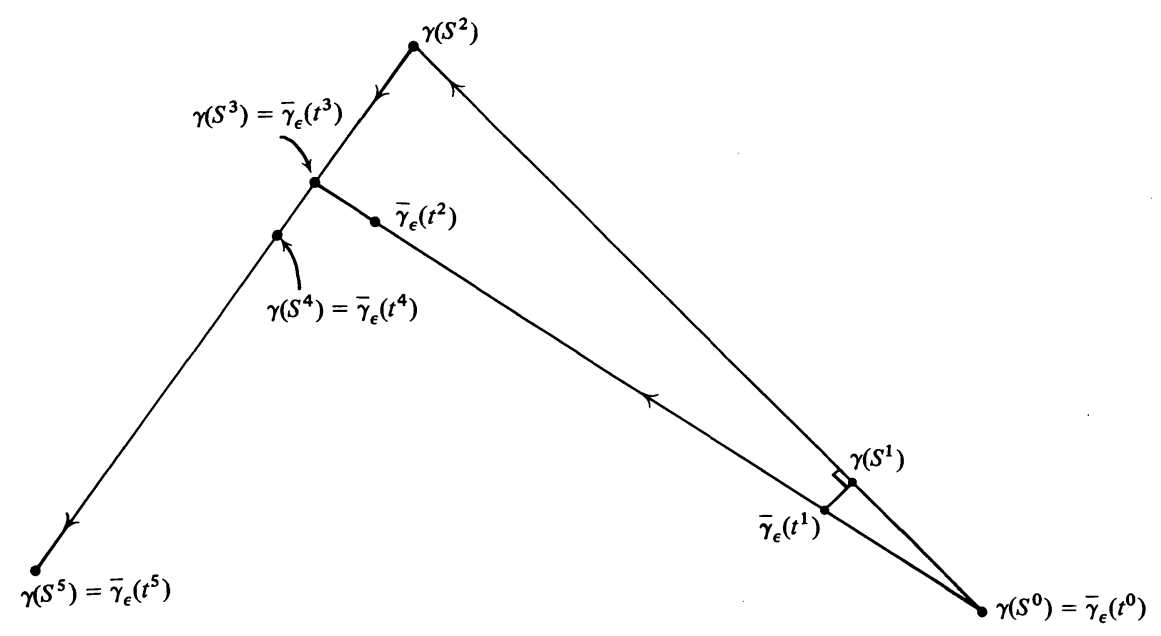

FIG. A.4

Choose $\bar{\varepsilon}<\frac{1}{2} d\left(\gamma, \bar{\gamma}_{\varepsilon}\left(\left[t^{1}, t^{2}\right]\right)\right)$ and $\bar{\varepsilon}$ less than half the distance between any pair of distinct points in $\left\{\gamma\left(s^{i}\right)\right.$ or $\left.\bar{\gamma}_{\varepsilon}\left(t^{i}\right) \mid i=0,1, \cdots, 5\right\}$ and such that $\bar{\varepsilon}$ is small for $\gamma$. Choose $\bar{\delta}$ much smaller than $\bar{\varepsilon}$ (as in Lemma A.1), and let $c_{\bar{\delta}}$ be an approximation to $\gamma$ as in Lemma A.1.

We now define $\bar{c}_{\delta}$.

For $t \notin\left[t^{0}, t^{5}\right]$ let $\vec{c}_{\delta}(t)=c_{\delta}(t)$. Thus for $t \notin\left[t^{0}, t^{5}\right]$ we have $d\left(\bar{\gamma}_{\varepsilon}(t), c_{\delta}(t)\right)$ $=d\left(\gamma(t), c_{\delta}(t)\right)<\bar{\delta}<\delta$.

For $t \in\left[t^{0}, t^{1}\right]$, let the curve $\bar{c}_{\delta}\left[t^{0}, t^{1}\right]$ be $c_{\delta}\left[s^{0}, s^{1}\right] \cup \bar{\tau}, \cdots, c_{\delta}\left(s^{1}\right)$ to $\bar{\gamma}_{\varepsilon}\left(t^{1}\right)$. By our choice of $\gamma\left(s^{1}\right)$ and Lemma A.1 this geodesic coincides with $\tau$ (but may be slightly shorter or longer). We choose the parameter on this segment of $\bar{c}_{\delta}$ (of course in $\left[t^{0}, t^{1}\right]$ ) to be proportional to arclength. By our choice of $\bar{\varepsilon}$ and $\bar{\delta}$ (and $\left.s^{1}, t^{1}\right)$ we have for all $t \in\left[t^{0}, t^{1}\right], d\left(\bar{c}_{\delta}(t), \gamma\left(\frac{i}{m}\right)\right)<\delta / 2$ and $d\left(\bar{\gamma}_{\varepsilon}(t), \gamma\left(\frac{i}{m}\right)\right)$ $<\delta / 4$ and hence $d\left(\bar{\gamma}_{\varepsilon}(t), \bar{c}_{\delta}(t)\right)<\delta$.

For $t \in\left[t^{1}, t^{2}\right]$, let $\bar{c}_{\delta}(t)=\bar{\gamma}_{\varepsilon}(t)$.

For $t \in\left[t^{2}, t^{4}\right]$ there are two cases. In the first case there is a $\bar{t} \in\left[t^{2}, t^{3}\right]$ and an $\bar{s} \in\left[s^{2}, s^{4}\right]$ such that $\bar{\gamma}_{\varepsilon}(t)=c_{\delta}(s)$. In this case, $\bar{c}_{\delta}\left[t^{2}, t^{4}\right]$ is the curve $\bar{\gamma}_{\varepsilon}\left[t^{2}, \bar{t}\right] \cup \bar{c}_{\delta}\left[\bar{s}, s^{4}\right]$ parameterized proportional to arclength. If the above does not happen, then $\bar{c}_{\delta}\left[t^{2}, t^{4}\right]$ is the curve $\bar{\gamma}_{\varepsilon}\left[t^{2}, t^{3}\right] \cup \sigma \cup c_{\delta}\left[s^{3}, s^{4}\right]$, where $\sigma$ is the minimal geodesic from $\gamma\left(s^{3}\right)$ to $c_{\delta}\left(s^{3}\right)$, and $\bar{c}_{\delta}\left[t^{2}, t^{4}\right]$ parameterized by arclength. In the above both cases we have

$$
d\left(\bar{\gamma}_{\varepsilon}(t), \bar{c}_{\delta}(t)\right) \leqslant d\left(\bar{\gamma}_{\varepsilon}(t), \bar{\gamma}_{\varepsilon}\left(\frac{i}{m}\right)\right)+d\left(\bar{\gamma}_{\varepsilon}\left(\frac{i}{m}\right), \bar{c}_{\delta}(t)\right)<\frac{\delta}{4}+\frac{\delta}{2}<\delta
$$

by choice of $\bar{\varepsilon}, \bar{\delta}, t^{i}$ and $s^{i}$. 
For $t \in\left[t^{4}, t^{5}\right]$, let $\bar{c}_{\delta}(t)=c_{\delta}(L(t))$, where $L(t)$ is the linear transformation from $\left[t^{4}, t^{5}\right]$ to $\left[s^{4}, s^{5}\right]$. In this case $d\left(\bar{\gamma}_{\varepsilon}(t), \bar{c}_{\delta}(t)\right)=d\left(\gamma(L(t)), c_{\delta}(L(t))\right)<\bar{\delta}$ $<\delta$.

The only thing left to check is that $\bar{c}_{\delta}$ is simple. To see this we note that $\bar{c}_{\delta}=\bar{\tau} \cup \bar{\gamma}_{\varepsilon}\left[t^{1}, \bar{t}\right] \cup c_{\delta}\left[\bar{s}, s^{1}\right]$ or $\bar{\tau} \cup \bar{\gamma}_{\gamma}\left[t^{1}, t^{3}\right] \cup \sigma \cup c_{\delta}\left[s^{3}, s^{1}\right]$. Each individual curve above is simple, and the fact that they only intersect at the endpoints follows from the choice of $\varepsilon$ and Remarks A.3.

Proof of Lemma 2.11. The fact that $\Omega_{1 / 2}^{m}(M)$ contains the simple closed piecewise geodesics $\gamma$ with $l_{i} \leqslant c$ and $\int_{M^{+}(\gamma)} K=\int_{M^{-}(\gamma)} K$ is clear. To see that $\Omega_{1 / 2}^{m}(M)$ is compact let $\left\{\gamma_{i}\right\}$ be a sequence in $\Omega_{1 / 2}^{m}(M)$. Since $\Omega_{1 / 2}^{m}(M) \subset$ $\Omega^{m}(M)$ and $\Omega^{m}(M)$ is compact, we may assume, by taking a subsequence, that $\gamma_{i} \rightarrow \gamma$ where $\gamma \in \Omega^{m}(M)$.

We need to show that $\gamma$ is nondegenerate and $\int_{M^{+}(\gamma)} K=\int_{M^{-}(\gamma)} K=2 \pi$. Since $M$ is compact its curvature is bounded and hence there is a number $V$ such that for all $i$, we have $\operatorname{Vol}\left(M^{+}\left(\gamma_{i}\right)\right)>V$ and $\operatorname{Vol}\left(M^{-}\left(\gamma_{i}\right)\right)>V$. Since $L\left(\gamma_{i}\right)$ (and $L(\gamma)$ ) are bounded (by $m c$ ), $\gamma_{i}$ has $m$ vertices, and the curvature of $M$ is positive, there is an $\varepsilon>0$ such that the volume of the tube of radius $\varepsilon$ about $\gamma_{i}$ (and $\gamma$ ) has volume less than $V / 2$. By Remarks A.3(a) if $c$ approximates $\gamma_{i}$ within $\varepsilon$ ( $c$ a simple closed piecewise smooth curve), we have $\operatorname{Vol}\left(M^{+}(c)\right)>V / 2$ and $\operatorname{Vol}\left(M^{-}(c)\right)>V / 2$. Let $c$ approximate $\gamma$ within $\varepsilon / 2$. Choose $i$ so large that $\gamma_{i}$ approximates $\gamma$ within $\varepsilon / 2$. Then $c$ approximates $\gamma_{i}$ within $\varepsilon$ so $\operatorname{Vol}\left(M^{+}(c)\right)>V / 2$ and $\operatorname{Vol}\left(M^{-}(c)\right)>V / 2$. Since this is true for all $\varepsilon / 2$ approximations to $\gamma$, we see that $\gamma$ is nondegenerate.

By Lemma 2.4 for each $i$ we can find a simple closed piecewise smooth curve $c_{i}$ such that $c_{i}$ approximates $\gamma_{i}$ within $1 / i$ and such that $2 \pi-1 / i<\int_{M^{+}\left(c_{i}\right)} K$ $<2 \pi+1 / i$. Now $c_{i} \rightarrow \gamma$ and $\lim _{i \rightarrow \infty} \int_{M^{+}\left(c_{i}\right)} K=2 \pi$. Since $\gamma$ is nondegenerate, Lemma 2.4 gives $\int_{M^{+}(\gamma)} K=2 \pi=\int_{M^{-}(\gamma)} K$. Hence $\gamma \in \Omega_{1 / 2}^{m}(M)$.

Proof of Lemma. 2.12. We first consider the case where $j \neq i+1$ and $j \neq i-1$.

By Lemma 2.10 (or 2.9 if $i$ is half free) there is an $\varepsilon_{i}>0$ such that for all $\varepsilon<\varepsilon_{i}$ there is a curve $\bar{\gamma}_{\varepsilon} \in \Omega^{m}(M)$ which differs from $\gamma$ only on $\left[\frac{i-1}{m}, \frac{i+1}{m}\right]$ and satisfies the conclusions of Lemma 2.10 (or 2.9). Let $S_{i}(\varepsilon)$ be the set between the curves $\gamma\left[\frac{i-1}{m}, \frac{i+1}{m}\right]$ and $\bar{\gamma}_{\varepsilon}\left[\frac{i-1}{m}, \frac{i+1}{m}\right]$ (i.e., the lying inside the ball of radius $2 c$ about $\gamma\left(\frac{i}{m}\right)$ ). By construction (see the proof of Lemma 2.9) we have $S_{i}(\varepsilon) \subset M^{+}(\gamma)$. On the other hand, by orientation considerations (Lemma A.4), we see that $S_{i}(\varepsilon) \subset M^{-}\left(\bar{\gamma}_{\varepsilon}\right)$. In fact, it is not hard to see that up to a set of measure 0 (i.e., $\gamma[0,1] \cup \bar{\gamma}_{\varepsilon}[0,1]$ ) we have $M^{+}\left(\bar{\gamma}_{\varepsilon}\right)=M^{+}(\gamma)-S_{i}(\varepsilon)$ and $M^{-}\left(\bar{\gamma}_{\varepsilon}\right)=M^{-}(\gamma) \cup S_{i}(\varepsilon)$. 
Similarly there is an $\varepsilon_{j}$ such that we can define $\overline{\bar{\gamma}}_{\varepsilon}$ and $S_{j}(\varepsilon)$ for all $\varepsilon<\varepsilon_{j}$. In this case, up to a set of measure 0 , we have $M^{+}\left(\overline{\bar{\gamma}}_{\varepsilon}\right)=M^{+}(\gamma) \cup S_{j}(\varepsilon)$ and $M^{-}\left(\overline{\bar{\gamma}}_{\varepsilon}\right)=M^{-}(\gamma)-S_{j}(\varepsilon)$.

Since $S_{j}\left(\varepsilon_{1}\right) \subset M^{-}(\gamma)$ and $S_{i}\left(\varepsilon_{2}\right) \subset M^{+}(\gamma)$ for $\varepsilon_{1}<\varepsilon_{j}$ and $\varepsilon_{2}<\varepsilon_{i}$, we have $S_{i}\left(\varepsilon_{1}\right) \cap S_{j}\left(\varepsilon_{2}\right)=\varnothing$. Since $M$ is convex $\int_{S_{i}\left(\varepsilon_{2}\right)} K>0$ and $\int_{S_{j}\left(\varepsilon_{1}\right)} K>0$, further, as $\varepsilon_{1}$ and $\varepsilon_{2}$ go to 0 , the integrals go to 0 .

Assume without loss of generality that $\int_{s_{i}\left(\varepsilon_{i}\right)} K>\int_{s_{j}\left(\varepsilon_{j}\right)} K$. Then for every $\varepsilon<\varepsilon_{j}$ there is an $\varepsilon_{1}<\varepsilon_{i}$ such that $\int_{s_{i}\left(\varepsilon_{1}\right)} K=\int_{s_{j}(\varepsilon)} K$. Now define $\gamma_{\varepsilon}$ by making an $\varepsilon$-deformation at $j / m$ and an $\varepsilon_{1}$-deformation at $i / m$. Since $j \neq i+1$, $j \neq i-1$, and $s_{i}\left(\varepsilon_{1}\right) \cap s_{j}(\varepsilon)=\varnothing$, we see that these two deformations can be made completely independent of each other. Hence by previous lemmas $\gamma_{\varepsilon}$ is a regular element of $\Omega^{m}(M), L\left(\gamma_{\varepsilon}\right)<L(\gamma)$, and if $i$ (or $j$ ) was half free with $\operatorname{Ext}(i)>A$ (or $-\operatorname{Ext}(j)>A$ ), then the same is true for $\gamma_{\varepsilon}$. It is also clear from the choice of $\varepsilon_{1}$ from $\varepsilon$ and from the fact that $M^{+}\left(\gamma_{\varepsilon}\right)=M^{+}(\gamma)-s_{i}\left(\varepsilon_{1}\right)+s_{j}(\varepsilon)$ (up to a set of measure 0) that $\int_{M^{+}\left(\gamma_{\varepsilon}\right)} K=2 \pi$. Hence $\gamma_{\varepsilon} \in \Omega_{1 / 2}^{m}(M)$.

The only thing left to show is that a similar construction can be made if $j=i+1$ or $j=i-1$. We assume without loss of generality that $j=i+1$.

Let $\varepsilon_{0}>0$ be so small that for all $0 \leqslant \varepsilon \leqslant \varepsilon_{0}$ the following hold:

(a) One can make the deformation of $\gamma$ at $i / m$ to a curve $\sigma_{\varepsilon}$ as in Lemma 2.10 or 2.9 .

(b) The exterior angle of $\sigma_{\varepsilon}$ at $j / m$ is negative.

(c) If $j$ is a half free-vertex of $\gamma$ such that $-\operatorname{Ext}(j)>A$, then we have $-\operatorname{Ext}_{\sigma_{e}}(j)>A$.

Let $K_{j} \subset M^{-}(\gamma)$ be the component which defines $j$ as a free (or half free) vertex of $\gamma$. By previous arguments we have $M^{-}(\gamma) \subset M^{-}\left(\sigma_{\varepsilon}\right)$, so we can choose the component $\bar{K}_{j} \subset M^{-}\left(\sigma_{\varepsilon}\right)$ such that $K_{j} \subset \bar{K}_{j}$. It is not hard to see that if $t \in\left[\frac{i}{m}, \frac{j+1}{m}\right]$ is a boundary parameter of $K_{j}$ for $\gamma$, then $t$ is a boundary parameter of $\bar{K}_{j}$ for $\sigma_{\varepsilon}$. In particular since $j$ was a free - vertex (respectively a half free - vertex) of $\gamma, j$ is a free (resp. half free) - vertex of $\sigma_{\varepsilon^{*}}$ (If $j$ was half free for $\gamma$, it could be free for $\sigma_{\varepsilon}$, but we still consider it to be half free in what follows.)

Let $f(\varepsilon)>0$ be such that for all $0 \leqslant \delta \leqslant f(\varepsilon)$ one can deform $\sigma_{\varepsilon}$ at $j / m$, as in Lemma 2.9 or 2.10 , to $\gamma_{\varepsilon}^{\delta}$. We can choose $f$ to be a continuous positive function on $\left[0, \varepsilon_{0}\right]$ this is clear from the proof of Lemma 2.9. Now consider the function

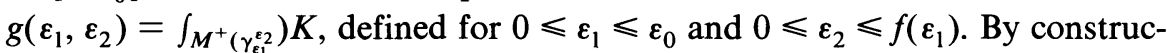
tion of $\gamma_{\varepsilon_{1}}^{\varepsilon_{2}}$ we see that $g\left(\varepsilon_{1}, \varepsilon_{2}\right)$ is continuous and $g(0,0)=2 \pi, g\left(0, \varepsilon_{2}\right)>2 \pi$ if $\varepsilon_{2}>0$, and $g\left(\varepsilon_{1}, 0\right)<2 \pi$ if $\varepsilon_{1}>0$. Since $g(0, f(0))>2 \pi$, there is an $\bar{\varepsilon}>0$ such that $g(\varepsilon, f(\varepsilon))>2 \pi$ for all $0 \leqslant \varepsilon \leqslant \bar{\varepsilon}$. Since $g(\varepsilon, 0) \leqslant 2 \pi$, for each $\varepsilon$ there is an $0 \leqslant \varepsilon_{1}(\varepsilon)<f(\varepsilon)$ such that $g\left(\varepsilon, \varepsilon_{1}\right)=2 \pi$. Thus it is easy to see that for $\varepsilon<\bar{\varepsilon}$, $\bar{\gamma}_{\varepsilon} \equiv \gamma_{\varepsilon}^{\varepsilon_{1}(\varepsilon)}$ will satisfy the conclusions of the lemma. 
Proof of Lemma 2.13. Let $\tau$ be as in the statement of the lemma, $\tau$ : $[0,1] \rightarrow M$ parameterized proportional to arclength. Let $V(t)$ be the unit vector perpendicular to $\tau^{\prime}(t)$ such that the orientation given by $\left(\tau^{\prime}(t), V(t)\right)$ is the orientation of $M$. Fix $\varepsilon>0$ and choose $\delta>0$ so small that the following hold:

(a) $F:[0,1] \times[-2 \delta, 2 \delta] \rightarrow M$ is a diffeomorphism where $F(t, s)=$ $\operatorname{Exp}_{\tau(t)}(s \cdot V(t))$

(b) If $\tau_{s}(t) \equiv F(t, s)$, then $L\left(\tau_{s}\right)<L(\tau)+\varepsilon$ for all $s \in[-\delta, \delta]$.

(c) $\delta<c / 4$.

We will let $\bar{\tau}_{s}$ represent the curve $\tau_{s}$ with the parameter proportional to arclength.

Let $m>\frac{1}{\delta}(L(\tau)+\varepsilon)$. Define $\gamma_{s}$ to be the closed piecewise geodesic curve such that $\gamma_{s\left[\frac{i}{m}, \frac{i+1}{m}\right]}$ is the minimizing geodesic from $\bar{\tau}_{s}\left(\frac{i}{m}\right)$ to $\bar{\tau}_{s}\left(\frac{i+1}{m}\right)$. We note that $d\left(\bar{\tau}_{s}\left(\frac{i}{m}\right), \bar{\tau}_{s}\left(\frac{i+1}{m}\right)\right) \leqslant \frac{1}{m} L\left(\tau_{s}\right)<\frac{1}{m}(L(\tau)+\varepsilon)<\delta<c$ for all $s \in[-\delta, \delta]$, thus the length of each geodesic segment $\gamma_{s\left[\frac{i}{m}, \frac{i+1}{m}\right]}$ is less than $c$.

Fix $s_{0} \in[-\delta, \delta]$. We now show that $\gamma_{s_{0}}$ is simple. Let $t_{k} \in[0,1]$ be such that $\tau_{s_{0}}\left(t_{k}\right)=\bar{\tau}_{s_{0}}(k / m)$. Since the sets $F_{k} \equiv F\left(\left(t_{k}, t_{k+1}\right) \times(-2 \delta, 2 \delta)\right)$ are disjoint, it is sufficient to show that $\gamma_{s_{0}\left(\frac{i}{m}, \frac{i+1}{m}\right)}$ is contained in $F_{i}$ for $i=0,1, \cdots, m-1$.

Since $d\left(\gamma_{s_{0}}(t), \tau\left(t_{i}\right)\right) \leqslant d\left(\gamma_{s_{0}}(t), \gamma_{s_{0}}\left(\frac{i}{m}\right)\right)+d\left(\gamma_{s_{0}}\left(\frac{i}{m}\right), \tau\left(t_{i}\right)\right)<2 \delta$ for $t \in$ $\left[\frac{i}{m}, \frac{i+1}{m}\right]$, we see that $\gamma_{s_{0}} \subset T \equiv F([0,1] \times(-2 \delta, 2 \delta))$.

Let $\sigma_{k}(s)=F\left(t_{k}, s\right)$ for $s \in[-2 \delta, 2 \delta]$. Now $T-\left(\sigma_{i}[-2 \delta, 2 \delta] \cup\right.$ $\left.\sigma_{i+1}[-2 \delta, 2 \delta]\right)$ consists of two connected components $F_{i}$ and $T-\operatorname{cl}\left(F_{i}\right)$ (the closure of $F_{i}$ ). Now since the geodesic segments $\gamma_{\left.s_{0} \mid \frac{i}{m}, \frac{i+1}{m}\right]}, \sigma_{i}$, and $\sigma_{i+1}$ all have length less than $c$, and since $\gamma_{s_{0}}\left(\frac{i}{m}\right)=\sigma_{i}\left(s_{0}\right)$ and $\gamma_{s_{0}}\left(\frac{i+1}{m}\right)=\sigma_{i+1}\left(s_{0}\right)$, we have that $\gamma_{s_{0}}\left(\left(\frac{i}{m}, \frac{i+1}{m}\right)\right)$ is disjoint from $\sigma_{i}$ and $\sigma_{i+1}$. Thus $\gamma_{s_{0}}\left(\left(\frac{i}{m}, \frac{i+1}{m}\right)\right) \subset F_{i}$ or $\gamma_{s_{0}}\left(\left(\frac{i}{m}, \frac{i+1}{m}\right)\right) \subset T-\operatorname{cl}\left(F_{i}\right)$. We need only show that $\gamma_{s_{0}}^{\prime}\left(\frac{i}{m}\right)$ points inward towards $F_{i}$. To see this let $W(t)$, for $t \in\left(\frac{i}{m}, \frac{i+1}{m}\right)$, be the unit tangent vector at $\bar{\tau}_{s_{0}}\left(\frac{i}{m}\right)$ which is tangent to the minimal geodesic from $\bar{\tau}_{s_{0}}\left(\frac{i}{m}\right)$ to $\bar{\tau}_{s_{0}}(t)$. Since $d\left(\bar{\tau}_{s_{0}}\left(\frac{i}{m}\right), \bar{\tau}_{s_{0}}(t)\right)<c, W(t)$ varies continuously with $t$. For $t$ near $\frac{i}{m}, W(t)$ points inwards towards $F_{i}$. For $t \in\left(\frac{i}{m}, \frac{i+1}{m}\right), W(t) \neq \sigma^{\prime}\left(s_{0}\right)$ and $W(t) \neq-\sigma^{\prime}\left(s_{0}\right)$, hence by continuity $W\left(\frac{i+1}{m}\right)$ points inwards towards $F_{i}$. Thus $\gamma_{s_{0}}\left(\frac{i}{m}, \frac{i+1}{m}\right) \subset F_{i}$, and $\gamma_{s_{0}}$ is simple.

So far we have shown that for $s \in[-\delta, \delta], \gamma_{s} \in \Omega^{m}(M)$ and is simple. Further $L\left(\gamma_{s}\right) \leqslant L\left(\tau_{s}\right)<L(\tau)+\varepsilon$. Thus we need only show that there is an $s_{0} \in[-\delta, \delta]$ such that $\int_{M^{+}\left(\gamma_{s_{0}}\right)} K=2 \pi$.

We have for all $t_{1}, t_{2} \in[0,1]$ that $d\left(\gamma_{\delta}\left(t_{1}\right), \tau\left(t_{2}\right)\right) \geqslant d\left(\bar{\tau}_{\delta}\left(t_{1}\right), \tau\left(t_{2}\right)\right)-$ $d\left(\gamma_{\delta}\left(t_{1}\right), \bar{\tau}_{\delta}\left(t_{1}\right)\right)>\delta-\delta=0$, hence $\gamma_{\delta}\left(t_{1}\right) \neq \tau\left(t_{2}\right)$, i.e., $\gamma_{\delta} \cap \tau=\varnothing$. Since $\gamma_{\delta}\left(\frac{i}{m}\right) \in M^{+}(\tau)$, we see that $\gamma_{\delta} \subset M^{+}(\tau)$, and by orientation considerations $M^{+}\left(\gamma_{\delta}\right) \subset M^{+}(\tau)$. Similarly $M^{+}(\tau) \subset M^{+}\left(\gamma_{-\delta}\right)$. 
Thus, by the convexity of $M, \int_{M^{+}\left(\gamma_{\delta}\right)} K<\int_{M^{+}(\tau)} K=2 \pi<\int_{M^{+}\left(\gamma_{-\delta}\right)} K$, and we can find an $s_{0} \in[-\delta, \delta]$ such that $\int_{M^{+}\left(\gamma_{s_{0}}\right)} K=2 \pi$. Hence $\gamma_{s_{0}} \in \Omega_{1 / 2}^{m}(M)$, and the proof is completed.

\section{References}

[1] M. S. Berger \& E. Bombieri, On Poincaré's isoperimetric problem for simple closed geodesics, preprint.

[2] A. L. Besse, Manifolds all of whose geodesics are closed, Ergebnisse Math. und ihrer Grenzgebiete, Vol. 93, Springer, Berlin, 1978.

[3] G. D. Birkhoff, Dynamical systems, Colloq. Publ. Vol. 9, Amer. Math. Soc., 1927.

[4] C. B. Croke \& A. Weinstein, Closed curves on convex hypersurfaces and periods of nonlinear oscillations, Invent. Math. 64 (1981) 199-202.

[5] S. Kobayashi \& K. Nomizu, Foundations of differential geometry, Vol. I, Interscience, New York, 1969.

[6] H. Poincaré, Sur les lignes géodésiques des surfaces convexes, Trans. Amer. Math. Soc. 6 (1905) 237-274.

University of Pennsylvania 